\title{
Hourly performance forecast of a dew point cooler using explainable Artificial Intelligence and evolutionary optimisations by 2050
}

(i) The corrections made in this section will be reviewed and approved by a journal production editor.

\author{
Yousef Golizadeh Akhlaghi ${ }^{\mathrm{a}}$, Koorosh Aslansefat ${ }^{\mathrm{b}}$, Xudong Zhao ${ }^{\mathrm{a}}$, Saba Sadati ${ }^{\mathrm{c}}$, Ali Badiei ${ }^{\mathrm{a}}$, Xin Xiao ${ }^{\mathrm{a}}$, Samson \\ Shittu ${ }^{\mathrm{a}}$, Yi Fan ${ }^{\mathrm{a}}$, Xiaoli Ma ${ }^{\mathrm{a}}$
}

${ }^{a}$ Centre for Sustainable Energy Technologies, Energy and Environment Institute, University of Hull, Hull HU6 $7 \mathrm{RX}, \mathrm{UK}$

${ }^{b}$ Department of Computer Science, University of Hull, Hull HU6 7RX, UK

'School of Mechanical, Aerospace and Civil Engineering, The University of Manchester, Manchester M13 9PL, UK

\begin{abstract}
1 Abstract
The empirical success of the Artificial Intelligence (AI), has enhanced importance of the transparency in black box Machine Learning (ML) models. This study pioneers in developing an explainable and interpretable Deep Neural Network (DNN) model for a Guideless Irregular Dew Point Cooler (GIDPC). The game theory based SHapley Additive exPlanations (SHAP) method is used to interpret contribution of the operating conditions on performance parameters. Furthermore, in a response to the endeavours in developing more efficient metaheuristic optimisation algorithms for the energy systems, two Evolutionary Optimisation (EO) algorithms including a novel bio-inspired algorithm i.e., Slime Mould Algorithm (SMA), and Particle Swarm Optimization (PSO), are employed to simultaneously maximise the cooling efficiency and minimise the construction cost of the GIDPC. Additionally, performance of the optimised GIDPCs are compared in both statistical and deterministic way. The comparisons are carried out in diverse climates in 2020 and 2050 in which the hourly future weather data are projected using a high-emission scenario defined by Intergovernmental Panel for Climate Change (IPCC). The results revealed that the hourly COP of the optimised systems outperform the base design. Although power consumption of all systems increases from 2020 to 2050, owing to more operating hours as a result of global warming, but power savings of up to $72 \%, 69.49 \%, 63.24 \%$, and $69.21 \%$ in hot summer continental, Arid, tropical rainforest and Mediterranean hot summer climates respectively, can be achieved when the systems run optimally.
\end{abstract}

Keywords: Dew point cooler; Multi objective evolutionary optimization; Particle Swarm Optimization; Slime Mould Algorithm; Artificial Intelligence

\section{Introduction}

Air conditioners are vital need of the modern buildings for providing comfortable indoor air for the residents. Several alternatives for the traditional coolers [1] are introduced to improve the cooling efficiency but among them, Evaporative Coolers (ECs) absorbed more attention owing to their intelligible structure and high efficiency [2]. ECs are categorized into two types, i.e., Direct Evaporative Coolers (DECs) and Indirect Evaporative Coolers (IECs) [5]. However, IECs are more favourable among the researchers due to their ability in delivering the cooled air (supply air) within the comfortable relative humidity ranges [7]. Continuous endeavours resulted in development of Dew Point Coolers 
(DPCs) which enables the supply air to reach the dew point temperature [8]. The main innovation of DPCs relies in invention of a M-cycle Heat and Mass Exchanger (HMX) which contributes towards increasing the efficiency of the ECs by up to $30 \%$ [7]. DPCs are categorized into two types based on direction of the air flows within the HMX, i.e., cross flow and counter flow [8]. In a project in USA, which is known as Coolerado ${ }^{\varpi}$, the cross flow DPC is tested for the first time in which the high efficiency of the DPC, i.e., wet bulb efficiency of $80 \%$, was proved [7]. This experiment followed by numerous research studies which were mainly aimed to assess the efficiency of the technology in more details. For instance, Jradi et al. [9], showed that in a cross flow DPC, the wet bulb efficiency of up to $117 \%$ is reachable when the supply air flow rate is in the range of $300-1500 \mathrm{~m}^{3} \cdot \mathrm{h}^{-1}$.

Continuous studies resulted in introduction of a state-of-the-art DPC in which the flat plates within HMX are replaced by corrugated plates [10]. This resulted in 30-60\% more cooling efficiency in the proposed counter flow DPC with Guideless Irregular HMX (GIDPC). Furthermore, it is concluded that the GIDPC can reach the record COP of 52.5 in the identified ideal condition in which the working air ratio was 0.36 [11]. In separate studies, the best operating conditions of a counter flow DPC [12] which led to improved performance in terms of wet bulb efficiency and COP is identified [13]. Badiei et al. [14], developed a whole building energy model for the GIDPC in which the performance of the system in different climates are compared by various models. It was concluded that the developed building energy model performs better than the selected numerical model.

The immense growth in data volume has enhanced the necessity of implementing the Machine Learning (ML) algorithms [15] to build the data-driven models for energy systems. The data-driven ML models for the DPCs are a vital need to integrate the embedded information within data through the ML algorithms. This can lead to commercialisation of this newly emerged technology and to improvement of the decision-making processes in future [16]. The concern in limiting the effect of global warming [17] and enhancing the efficiency of the technology are mainly mitigated through technological breakthrough which has led to underrated importance of optimisation algorithms. In general, the optimisation is aimed to reduce the construction and operating costs simultaneously [18]. Owing to the numerous operating, design and performance parameters in the energy systems, the metaheuristic algorithms [19] are extensively considered to overcome the complexity of the systems by exploring all possible solutions by considering one or multiple objective functions.

In one of the pioneer studies, the Response Surface Methodology (RSM) is used for a cross flow DPC [20] in which, it is concluded that the supply air flow rate, and air properties of the intake air are the main parameters affecting the system performance. However, the study suffers from lack of optimisation algorithm to truly identify the optimum parameters. In addition, the selected parameters are limited to a few operating parameters for a traditional DPC. The Multi Objective Optimization (MOO) and Group Method of Data Handling-type neural network (GMDH) are employed [21] to disclose the performance improvement when a cross flow DPC operates under the optimum conditions which led to $8.1 \%$ and $6.9 \%$ improvements in COP and cooling capacity respectively. However, the decision variables and objective functions were limited to few parameters and the annual average amounts were considered rather than hourly data which could lead to more sensible outcomes. Although the hourly optimisation is considered in another study by Sohani et al. [22], but the study was again limited to only two decision variables. Although a through operating and design parameters are considered in a regression-based study for a counter flow DPC [23] but the study was ignored the optimisation algorithms. The optimum length $(0.50 \mathrm{~m})$ and working air ratio (0.40) are identified for a DPC in an optimization-based study [24]. However, the study was ignored the applicability of the outcomes in diverse climates as well as the hourly performance investigation of the optimised system.

The aforementioned ML studies have mainly focused on the existed commercial counter flow and cross flow DPCs but there are few ML based studies for the novel GIDPC. A regression-based studies are conducted in energy systems [25] in which for the GIDPC, Akhlaghi et al. [26], used Multiple Polynomial Regression (MPR) to provide the polynomial equation for performance prediction of the GIDPC. However, the model was unable to consider the design parameters in the produced equations. In addition, in another study [27], the Feedforward Neural Network (FFNN) and Genetic Algorithm (GA) are developed for the GIDPC to predict the performance of the optimized system in diverse climates. The results revealed that the COP and surface area of the system can be improved by up to $72.5 \%$ and $23.57 \%$ respectively [27]. However, the study has ignored the hourly performance of the system and the saving values were based on the monthly average climate data which can lead to substantially inaccurate results. 
Although the valuable results are presented in the reviewed literature but there are outstanding gaps in them. Firstly, the majority of the studies are based on the traditional M-cycle DPCs whereas the GIDPC is proved to have a superior efficiency [11]. Secondly, the optimisation-based studies have focused on Genetic Algorithm (GA) and they suffer from comparing diverse algorithms to establish the best one. However, the most substantial gap is the lack of performance investigation in upcoming years when the proposed systems will operate in diverse unforeseen climates. This can present the efficiency of the optimisation outcomes by disclosing the energy saving potential of the GIDPC in forthcoming operating climates. In addition, the aforementioned developed models are considered as the black-box ML models which are suffering from a barrier of explainability. An emerging need in interpreting the sophisticated ML models such as opaque Deep Neural Networks (DNNs) which empirically have been successful over the last years, has become vital [28].

Therefore, firstly, this research is pioneered in bringing eXplainable Artificial Intelligence (XAI) to the GIDPC research studies by employing the game theory based SHapley Additive exPlanations (SHAP) method. For the newly evolving $\mathrm{XAI}$, this is considered as a preliminary approach by SHAP to demonstrate the features contributions on the predicted parameters throughout the force plots. The XAI is based on the Deep Neural Network (DNN) model which was developed by the authors [27]. Secondly, it is aimed to employ the state-of-the-art bio-inspired algorithm, i.e., Slime Mould Algorithm (SMA) as the main MOO method along with the popular Particle Swarm Optimization (PSO) to identify the optimum operating and design parameters of the system by comparing the performance of both methods. The decision variables of the algorithms are: velocity of the intake air, working air fraction, height, channel gap, and number of layers in the HMX structure. Both algorithms are aimed to maximise the cooling efficiency of the system and to minimise the construction cost. Eventually, a climate scenario defined by Intergovernmental Panel for Climate Change (IPCC) is used to produce the hourly temperature and relative humidity in 2020 and 2050 for the performance prediction of the GIDPC in upcoming climate [29]. Eventually, the power consumption of the systems, i.e., base system, optimized system by PSO and optimized system by SMA, in operating hours throughout a year are calculated and the power saving potential of the GIDPC is presented in both 2020 and 2050. The summarised research methodology of the current study is shown in Fig. 1.

Fig. 1

Research methodology

A developed explainable and interpretable DNN model using SHAP A game theory based SHapley Additive exPlanations (SHAP) method is used to interpret contribution of operating/design parameters on performance parameters

Optimisation of the GIDPC using SMA and PSO methods The SMA is used as a novel metaheuristic algorithms and the PSO is used as a wellknown swarm-based metaheuristic method

Power saving potential of the optimised systems considering climate change The hourly performance of the optimised GIDPCs is compared in both statistical and deterministic way with the base system in the selected climates to predict the power saving potential of the optimised systems in 2020 and 2050

Summary of the research methodology.

\section{Guideless Irregular dew point cooler}

The GIDPC as a novel counter-flow DPC with guideless irregular HMX [10] is selected in the current study. The selected GIDPC is called the base system in this study which its dimensions are inspired from authors previous study and listed in the upcoming sections of the current study [27]. The GIDPC is constructed from few components in which the HMX is the core section which is depicted in Fig. 2(a). As seen, the considered HMX is constructed from plenty corrugated layers which have formed several dry and wet channels to carry out the cooling and evaporation 
processes during the system operation. The utilization of corrugated layers as a replacement of the traditional flat layers result in more heat transfer area and less flow resistance within the channel [10]. As seen in Fig. 2(b), two wet sides of neighbour layers form the wet channels and two dry sides of the neighbour layers build the dry channels. The material used in wet sides is Coolmax-fabric while the dry sides are built from aluminium.

\section{Fig. 2}
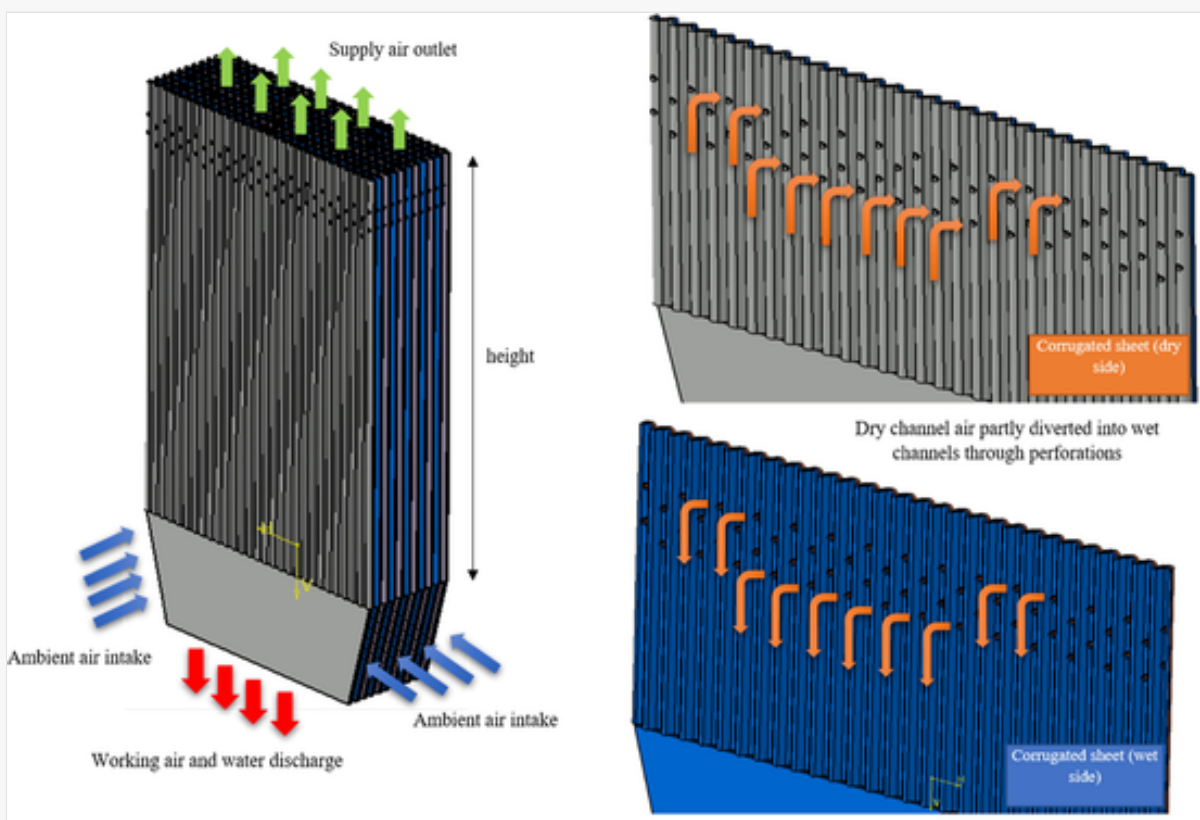

(a)

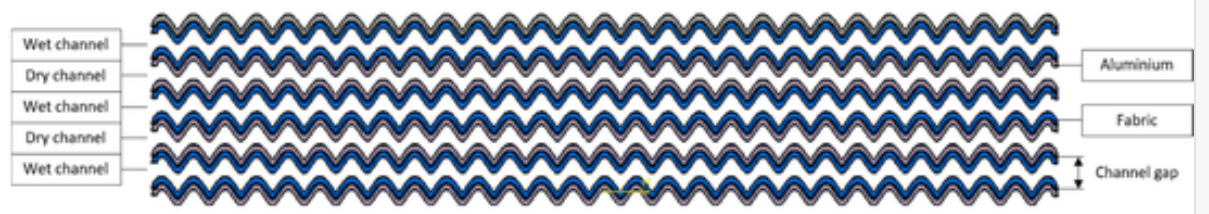

(b)

Heat and mass exchanger (a): heat and mass exchanger structure (b): corrugated surfaces (top view) [27].

To cool down the air, firstly the intake air with determined temperature and relative humidity enters the dry channel with adjusted velocity. According to value of the adjusted working air ratio, part of the intake air is transferred into the wet channel, i.e., called working air, via the holes located on the layers and the rest of it is delivered into the space as the cooled supply air. The temperature dropping of the intake air happens while it moves along the dry channel by losing the heat to the neighbour wet channel. This occurs owing to the cooler surface and the water evaporation taking 
place within wet surface of the wet channel. The working air within the wet channel receives considerable amount of heat and moisture form the wet surface and carries them out as the warm and wet working air (also called exhaust air).

\section{Explainable Artificial Intelligence}

\subsection{Deep Neural Network}

The developed DNN model is chosen for the optimization and interpretation of the features contributions in XAI. Neural Networks (NNs) are black box models which have three main sections: (1) input layer, (2) hidden layer(s), (3) output layer. The input layer includes the input variables of the model which contains the key operating and design parameters of the GIDPC for the developed DNN model [27]. These parameters are temperature, relative humidity and velocity of the intake air, and working air fraction which are all representing the operating parameters while the design parameters are: height of the HMX, channel gap and number of layers in the HMX. The output layer includes those performance parameters of the GIDPC which are needed to be predicted under various operating conditions. The output layer of the developed model includes the performance parameters of the GIDPC [30]. In the developed model, the cooling capacity, COP, wet bulb and dew point efficiency, temperature drop and surface area of the layers are the performance parameters. Among them, the cooling capacity and COP are used to observe the system performance in terms of cooling and power consumption. However, to evaluate the system performance in more details, other parameters are needed as well. For instance, Wet-bulb efficiency is needed to see the system performance in reducing the intake air temperature to its wet-bulb temperature. In a similar manner, the dew point efficiency is used to observe the system performance in reducing the intake air temperature to its dew point temperature. In addition, to take the construction cost of the GIDPC into consideration, the surface area of the layers is selected as another performance parameter. The formulas for the selected performance parameters are listed in Table 1.

Table 1

(i) The table layout displayed in this section is not how it will appear in the final version. The representation below is solely purposed for providing corrections to the table. To preview the actual presentation of the table, please view the Proof.

Performance parameters of the GIDPC.

\begin{tabular}{|c|c|}
\hline Performance parameter & Formula \\
\hline Cooling capacity & Qcooling =CpTdry,in-Tdry,out(1- $\varphi)$ Qm,dr,in \\
\hline $\mathrm{COP}$ & $\mathrm{COP}=\frac{\mathrm{Q}_{\text {cooling }}}{\mathrm{W}_{\text {fan }}+\mathrm{W}_{\text {pump }}}$ \\
\hline Wet bulb efficiency & $\varepsilon_{\mathrm{wb}}=\frac{\mathrm{T}_{\mathrm{dry}, \mathrm{in}}-\mathrm{T}_{\mathrm{dry}, \mathrm{out}}}{\mathrm{T}_{\mathrm{dry}, \mathrm{in}}-\mathrm{T}_{\mathrm{dry}, \mathrm{in}, \mathrm{wb}}}$ \\
\hline Dry bulb efficiency & $\varepsilon_{\mathrm{dp}}=\frac{\mathrm{T}_{\mathrm{dry}, \mathrm{in}}-\mathrm{T}_{\mathrm{dry}, \text { out }}}{\mathrm{T}_{\mathrm{dry}, \mathrm{in}}-\mathrm{T}_{\mathrm{dry}, \mathrm{in}, \mathrm{dp}}}$ \\
\hline Surface area & $\mathrm{As}=(\mathrm{NL}) \mathrm{Hw}$ \\
\hline \multicolumn{2}{|l|}{ Terms descriptions } \\
\hline \multicolumn{2}{|c|}{$\begin{array}{l}\mathrm{C}_{\mathrm{p}} \text { is specific heat capacity, } \mathrm{T}_{\mathrm{dry}, \text { in }} \text { is intake air temperature in dry channel, } \mathrm{T}_{\mathrm{dry}, \text { out }} \text { is outlet air temperature in dry channel, } \varphi \text { is } \\
\text { working air fraction, } \mathrm{Q}_{\mathrm{m}, \mathrm{dr} \text {,in }} \text { is mass flow rate of intake air in dry channel, } \mathrm{W}_{\text {fan }} \text { is electrical power consumed by fan, } \mathrm{W}_{\text {pump }} \text { is } \\
\text { electrical power consumed by pump, } \mathrm{T}_{\mathrm{dry}, \text { in,wb }} \text { is the wet-bulb temperature of the intake air in dry channel, } \mathrm{T}_{\mathrm{dry}, \text { in,dp }} \text { is the dew point } \\
\text { temperature of the intake air in dry channel, } A_{s} \text { is the surface area, } N_{L} \text { is the number of layers, } H \text { is height of the HMX and w } \\
\text { renresents the width of the surface }\end{array}$} \\
\hline
\end{tabular}

A big dataset with 78,125 data points is constructed using the validated numerical model [27] and considering the aforementioned operating, design and performance parameters. The holdout cross-validation is employed to split the big dataset into three subsets i.e., a training data set (70\%), a validation data set (15\%), and a testing data set (15\%). Fig. 3 , depicts the operating ranges of the inputs and the structure of the developed DNN model. The connections are weighted by initial random values which are needed to be updated through an iterative back propagation process. The 
back propagation is applied to update the weights with the aim of maximising the model accuracy [31]. The random constant bias values are also used to calibrate the outputs of each weighted sum of the inputs as well as to smooth the fitting process of the model. The hyperbolic tangent sigmoid function is selected as the activation function in each layer as a conclusion of a robust comparison with different functions. The structure of the DNN model is defined by selecting the number of layers and neurons. These two hyperparameters are calibrated through a test harness. Numerous DNN structures are built by monitoring the model quality through two accuracy metrics (Mean Square Error, (MSE), and coefficient of determination $\left(\mathrm{R}^{2}\right)$ ). The gradual complexity enhancement of the model was revealed that a model with two hidden layers and 45 neurons in each hidden layer were the most desirable DNN. The MSE of the selected model had the low value of 0.04 and $\mathrm{R}^{2}$ held the ultimate value of 1 .

Fig. 3

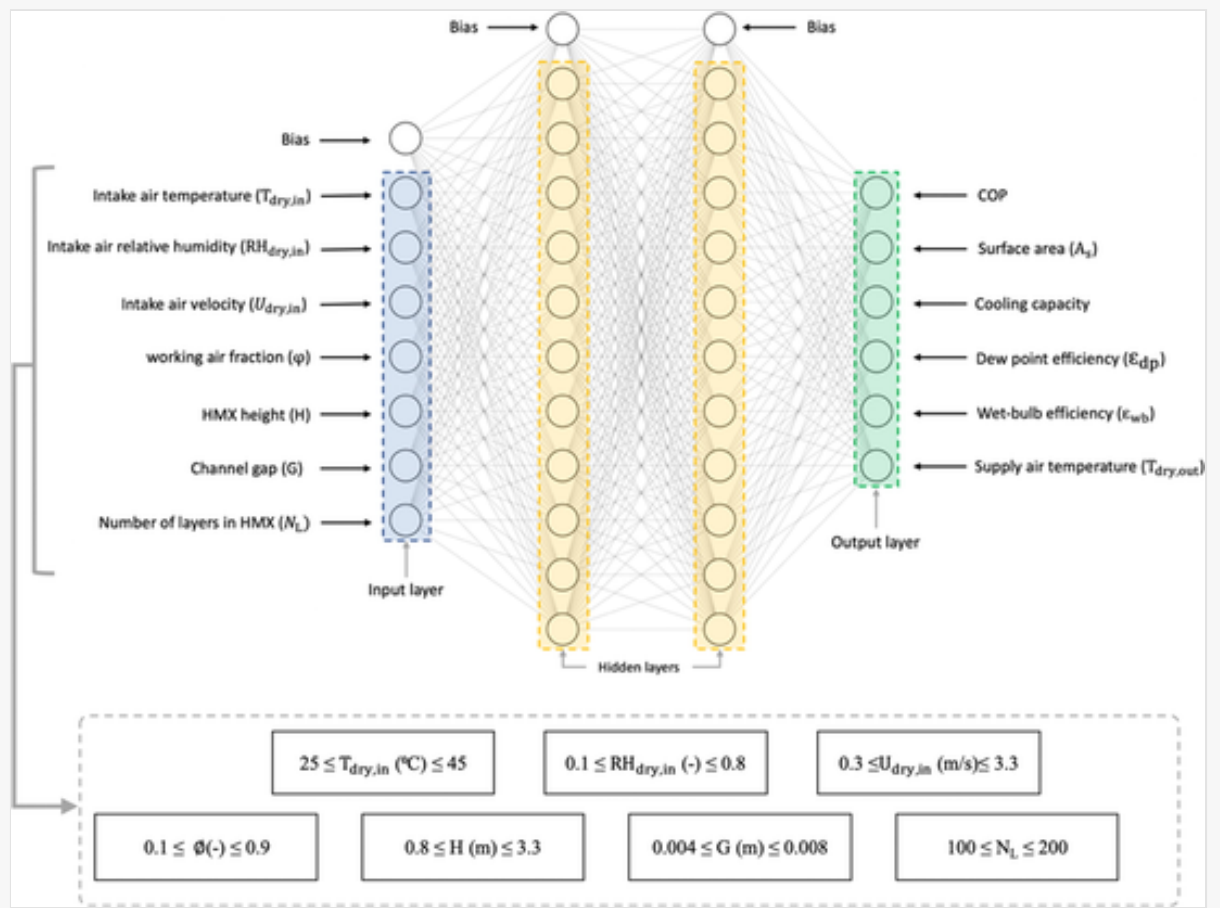

Structure of Deep Neural Network model and operating ranges of inputs for the GIDPC.

\subsection{Explainable Artificial Intelligence}

Owing to the empirical success of the ML models in complex computational tasks, the need for interpreting the black box models, e.g., a complex DNN model with numerous layers and parameters is increased [32]. The transparency of such models will contribute the experts with providing more detailed information about the model than a final accurate prediction [33]. Furthermore, it will lead to more trustable ML models in which the researchers can observe the evolving process of the model [32]. The aforementioned need for interpreting the complex ML models has led to appearance of eXplainable Artificial Intelligence (XAI) field. The newly evolving XAI has the ability to demonstrate the rationality of the results bin different ways [34]. SHapley Additive eXplanations (SHAP) is based on a game theory which can be used as a XAI method to interpret the ML models [35]. In this study, the SHAP is used to mainly show how the operating and design parameters of the GIDPC affect the performance parameters using the function provided by the developed DNN model. SHAP can demonstrate the contribution of each input to the predicted value using the calculated Shapley values which reveal how to distribute the predicted values (performance parameters) among the features (operating and design parameters). In SHAP, the Shapley value explanation is provided as an additive feature contribution [35]. In summary, SHAP describes the following three attributes:

1) Local accuracy:

$$
f(x)=g\left(\begin{array}{c}
\hat{A} \\
x
\end{array}\right)=\varphi_{0}+\sum_{i=1}^{N} \varphi_{i}^{\hat{A} x_{i}}
$$




$$
\text { if } x_{i}^{\hat{A}}=0 \text { then } \varphi_{i}=0
$$

\section{3) Consistency:}

For any two different models $f$ and ${ }_{f}^{\hat{A}}$ :e

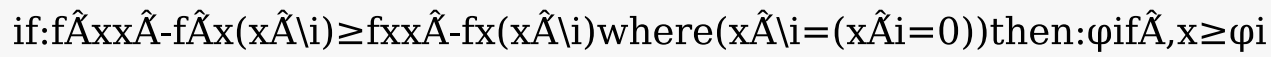

where $g$ represents the explanation model, $h$ is the mapping function, ${ }_{x}^{\hat{A}}$ represents the coalition vector, $\varphi_{i}$ is the feature attribution for the input $i, N$ is the maximum coalition size, $\hat{f}_{x}$ and $f_{x}$ are the mapping functions which use the simplified input $\underset{x}{\hat{A}}$ to map the original input $x$.

In local accuracy, the requirement of the explanation model $(\mathrm{g})$ which can match the main model (f) for the simplified input $\left(\begin{array}{l}\hat{A} \\ x\end{array}\right)$, is explained. The missingness simply says the contribution of missing input (feature) is zero. The consistency indicates when the model changes, the contribution of the feature will be higher and will stay same. Shapley values are given by the following expression which is the only set of values that satisfy the three aforementioned conditions:

$$
\varphi_{i}(f, x)=\sum_{\substack{\hat{A} \hat{A} \\
z \subseteq x}} \frac{|\hat{A}| z \mid !(N-|\hat{A}|-1) !}{N !}\left[f_{x}\left(\begin{array}{l}
\hat{A} \\
z
\end{array}\right)-f_{x}\left(\begin{array}{l}
\hat{A} \\
z \backslash i
\end{array}\right)\right]
$$

in which the $\left|\begin{array}{l}\hat{A} \\ z\end{array}\right|$ in the group of non-zero values in $\underset{z}{\hat{A}}$, as the subset of $\frac{\hat{A}}{x}$.

The Shapley values can be visualized as forces to demonstrate the attribution of each feature on the prediction [35,36]. Fig. 4 shows the force plots for each of the performance parameters in the developed DNN. Force plots show how each of the input variables (features) contribute to the value of the performance parameters. Each figure consists of two sub figures in which the first one is demonstrated for one representative data point (operating condition) and the second one is demonstrated for 10 data points (out of 78,125 data points used in DNN model) which are rotated 90 degrees and stacked together horizontally [38]. 


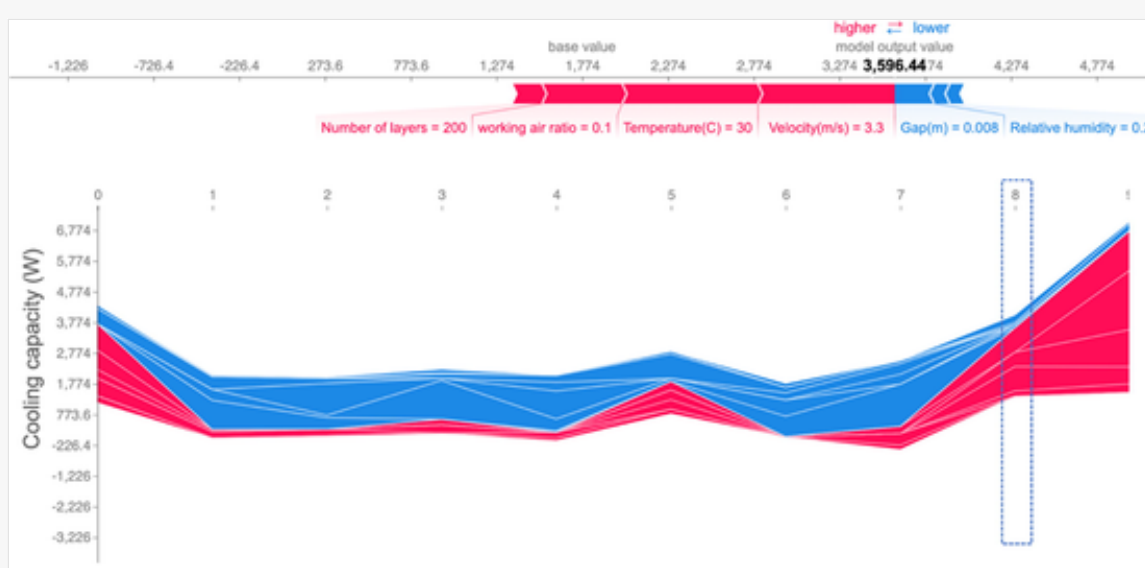

(a)
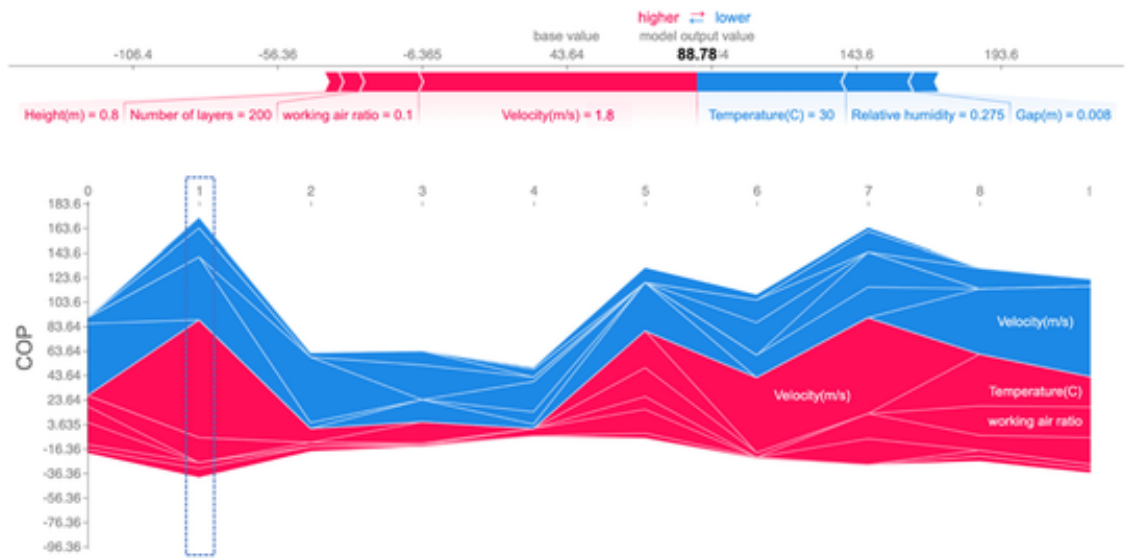

(b)

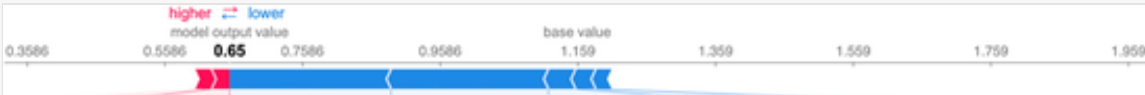

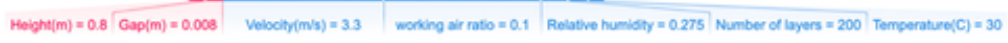

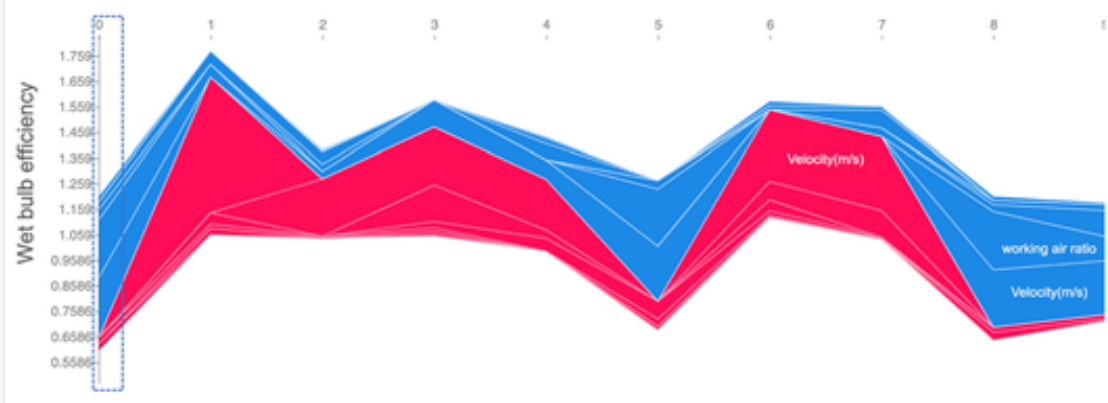

(c)
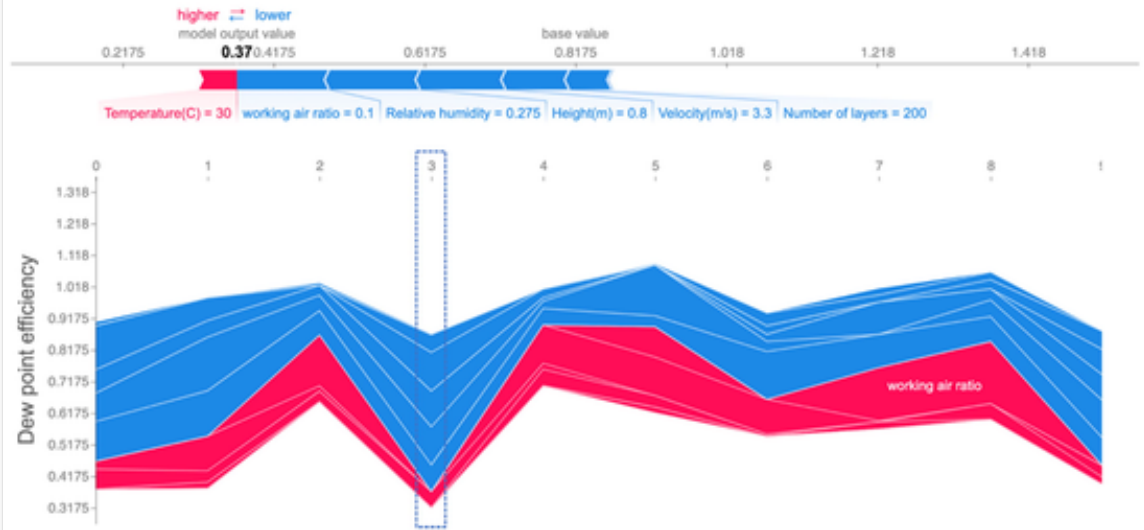

(d) 


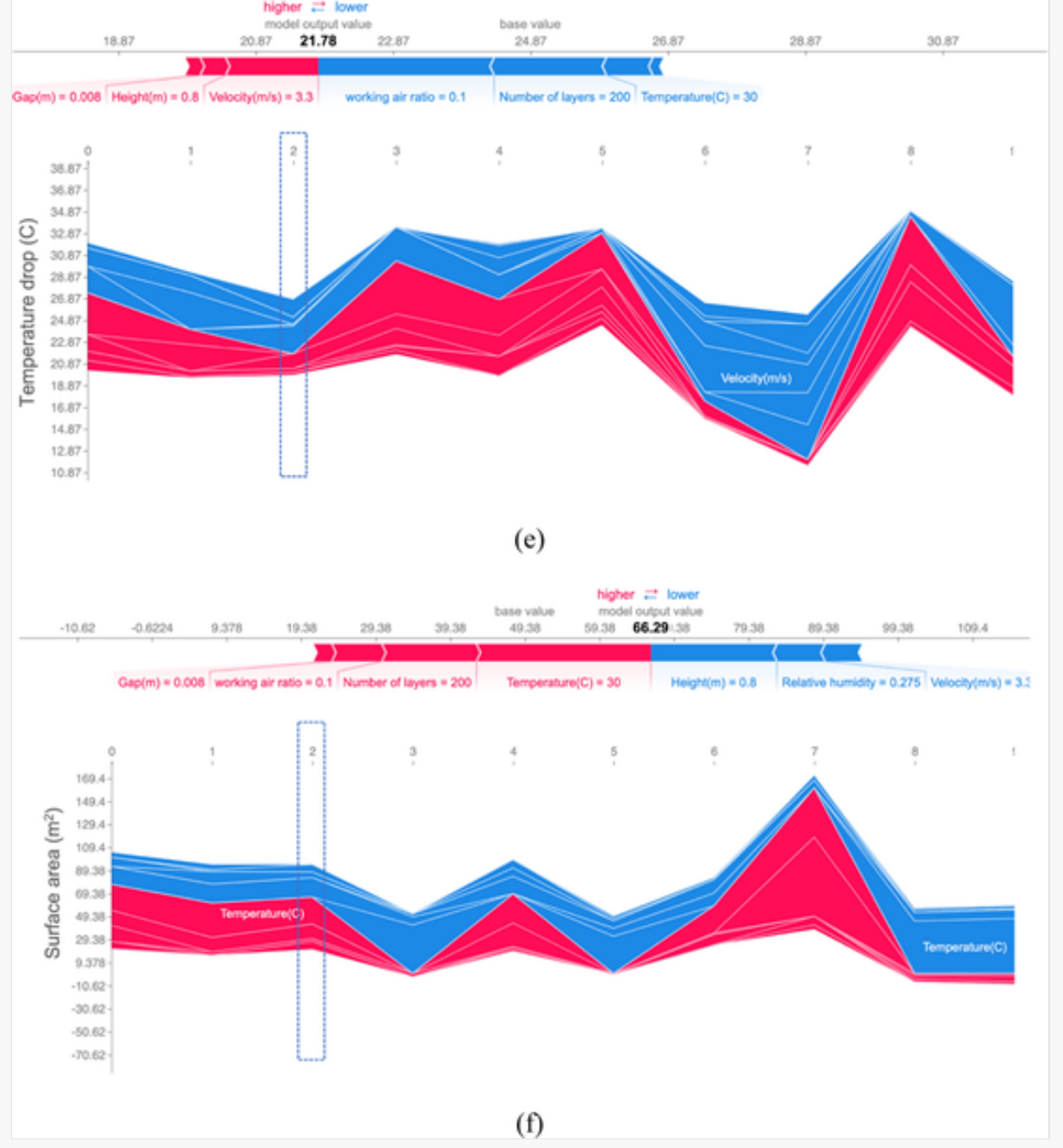

Force plots: (a): cooling capacity; (b): COP; (c): wet bulb efficiency; (d): dew point efficiency; (e): temperature drop; (f) surface area.

Each feature is considered as a force which either has a positive or negative effect. The prediction for each performance parameter starts from the base value which is the average value of the performance parameter in the training dataset used to train the DNN which would happen if the effect of features (operating and design parameters) was not considered. There is also an output value in each figure which is identical to the predicted value by the DNN model. SHAP values are the arrows in red and blue indicating how much contribution each feature has on the output value which results in increasing or decreasing the prediction. There are positive contributions in red colours which mean that the correspondent feature contributes to increase the performance parameter value from the base value to the actual predicted value by the DNN whereas the negative contributions with blue colours contribute to decrease the value of 
the performance parameter from the base value to the actual predicted value. As seen, the contribution of each data point is different which is simply because of the different operating and design parameter values. These plots allow us to know the impact of each parameter on the predicted values in the desired data point. This information let the researchers to observe the positive and negative contribution of each parameter and identify the appropriate operating condition for each performance parameter. This will eventually result in improved system performance and energy management. For instance, Fig. 4 (a) shows the force plots for the cooling capacity in which the data point number 8 is selected as the representative. As seen, the base value is increased from $1.77(\mathrm{~kW})$ to the model predicted value of 3.59 $(\mathrm{kW})$ in which four inputs in red colours have positive contribution while other three inputs i.e., gap, relative humidity and height have negative contributions. The force plots of other performance parameters are also provided for ten sample data points as demonstrated in Fig. 4 (b)-(f).

\section{Proposed multi objective optimization methods: SMA and PSO}

The novel Slime Mould Algorithm (SMA) method is used to identify the optimum operating and design parameters of the GIDPC, in four selected operating climates, to improve the system efficiency in terms of cooling, power consumption and construction cost. Evolutionary Algorithms (EA) and intelligent swarm techniques are two major classifications of the metaheuristic algorithms. The common Genetic Algorithm (GA) falls into the group of EA while the algorithms like PSO are in the class of swarm intelligence techniques which are inspired from collective behaviour of some biological creatures. Exploration and exploitation are two stages of the metaheuristic algorithms which are needed to be balanced [39].

The No-Free-Lunch (NFL) theory [40] has proven that none of the substantially improved metaheuristic algorithms, can assure to find the global optimum. As a result, the endeavours in introducing the new efficient algorithms are in place in which the SMA is one of the newly introduced methods. The SMA makes a superb balance between the explorations and exploitations which leads to better statistical performance than the PSO. In addition, SMA is fast in operation and also in diverging to an optimal solution and has less computational complexity [41]. In addition, the Particle Swarm Optimization (PSO) is also developed to compare the results of two methods and to show the validity of the novel SMA]

\subsection{Slime Mould Algorithm}

The SMA, as one of the state-of-the-art bio-inspired algorithms that has been proposed by [41] and can diverge to the optimal value of the problem, is used as the first optimization algorithm. The SMA has three phases including approach food, wrap food and grabble food. In the approach food, Eq. (5) can be used as a mathematical model for approaching behaviour of slime mould.

$$
\overrightarrow{X(t+1)}=\left\{\begin{array}{c}
\overrightarrow{X_{b}(t)}+\overrightarrow{v b} \cdot\left(\vec{W} \cdot \overrightarrow{X_{A}(t)}-\overrightarrow{X_{B}(t)}\right), r<\tanh |S(i)-D F| \\
\overrightarrow{v c} \cdot \overrightarrow{X(t)}, r \geq \tanh |S(i)-D F|
\end{array}\right.
$$

where $|S(i)-D F|$ stands for the distance between the fitness of $\vec{X}$ and the fitness achieved in all iterations $(i \in 1,2, \cdots, n)$, $\overrightarrow{v b}$ is an internal vector in SMA with the range defined based on Eq. (6), $\overrightarrow{v c}$ is another vector that linearly decreases from one to zero, $t$ stands for current iteration number. The location of a point with the highest rate of odour concentration that currently found can be represented by $\overrightarrow{X_{b}}$, and current location of the slime mould is represented by $\vec{X}$. In each iteration, two individuals are randomly selected from the swarm as $\overrightarrow{X_{A}}$ and $\overrightarrow{X_{B}}$ and their distance will be gained by weight of slime mould as $\vec{W}$.

$$
\overrightarrow{v b}=\left[-\operatorname{arctanh}\left(-\left(\frac{t}{\max \_t}\right)+1\right), \operatorname{arctanh}\left(-\left(\frac{t}{\max \_t}\right)+1\right)\right]
$$

Weight of slime mould $\vec{W}$ can be calculated by Eq. (7) in which "Smell Index" is a sorted version of fitness vector. 


$$
\overrightarrow{W(\text { SmellIndex }(i))}=\left\{\begin{array}{c}
1+r \cdot \log \left(\frac{b F-S(i)}{b F-w F}+1\right), \text { condition } \\
1-r \cdot \log \left(\frac{b F-S(i)}{b F-w F}+1\right), \text { others }
\end{array}\right.
$$

where $r$ is a uniformly distributed random number with the range of $[0,1]$, the ranked first half of the population is indicated by $S(i), \mathrm{bF}$ and $\mathrm{wF}$ denote the best and the worst fitness values in each iteration respectively. In the wrap food phase, the location of the slime mould can be formulated using the Eq. (8) as follows:

$$
\overrightarrow{X^{*}}=\left\{\begin{array}{c}
\text { rand } \bullet(U B-L B)+L B, \text { rand }<z \\
\overrightarrow{X_{b}(t)}+\overrightarrow{v b} \cdot\left(W \cdot \overrightarrow{X_{A}(t)}-\overrightarrow{X_{B}(t)}\right), r<p \\
\overrightarrow{v c} \cdot \overrightarrow{X(t)}, r \geq p
\end{array}\right.
$$

where upper bound and lower bound of the search algorithm can be defined by UB and LB, respectively. Both rand and $\mathrm{r}$ are uniformly distributed random numbers which range from 0 to 10 .

In the grabble food phase which is generated through a random oscillation procedure with the bound of $[-a, a]$, and a negative gain, $\overrightarrow{v b}$ which gradually tends to zero over the iterations. Similarly, $\overrightarrow{v c}$ value is generated through a random oscillation procedure with the bound of $[-1,1]$ and a negative gain that eventually approaches zero in the final iteration. The initial parameters for the SMA are listed in Table 2.

\section{Table 2}

(i) The table layout displayed in this section is not how it will appear in the final version. The representation below is solely purposed for providing corrections to the table. To preview the actual presentation of the table, please view the Proof.

Parameter settings for SMA.

\begin{tabular}{|l|l|}
\hline Parameter & \multicolumn{1}{l|}{ Values } \\
\hline Max. number of iterations & 500 \\
\hline Slime mould dimension size & 5 \\
\hline Number of search agents & 30 \\
\hline Upper Bound Vector (UB) & {$[3.3,0.9,3.0,0.008,200]$} \\
\hline Lower bound vector (LB) & {$[2.0,0.1,0.8,0.004,100]$} \\
\hline Degradation ratio & 0.1 \\
\hline
\end{tabular}

The optimization function is developed based on the fitness function and the constraint function. The fitness function is the trained DNN model which is used to approach the optimisation objectives while the constraint function limits the operation of the fitness function through the predefined operating ranges. The following Multi-Objective (MO) fitness function is considered in the SMA in which seven dimensions are associated, i.e., intake air temperature $\left(\mathrm{T}_{\mathrm{dry}}\right.$,in $)$, intake air relative humidity $\left(\mathrm{RH}_{\mathrm{dry}, \text { in }}\right)$, intake air velocity $\left(U_{\text {dry,in }}\right)$, working air ratio $(\varphi)$, HMX height $(\mathrm{H})$, gap $(\mathrm{G})$, and number of layers in HMX structure $\left(\mathrm{N}_{\mathrm{L}}\right)$. Noted that $T_{d r y, i n}$ and $R H_{d r y, i n}$ are predefined based on the climates. It means that the decision variables of the optimization algorithm are intake air velocity, working air ratio, channel gap and number of layers. In addition, the objective of the optimization is to maximise the cooling capacity $\left(Q_{\text {cooling }}\right)$, COP and wet bulb efficiency $\left(\varepsilon_{w b},\right)$, and to minimise the surface area of the layers $\left(A_{s},\right)[27]$.

$$
\mathrm{J}\left(\mathrm{T}_{\mathrm{dry}, \mathrm{in}}, \mathrm{RH}_{\mathrm{dry}, \text { in }}, U_{\mathrm{dry}, \text { in }}, \varphi, \mathrm{H}, \mathrm{G}, \mathrm{N}_{\mathrm{L}}\right)=\mathrm{W}_{1} \frac{\mathrm{Q}_{\text {cooling }}}{\mathrm{RQ}_{\text {cooling }}}+\mathrm{W}_{2} \frac{\mathrm{COP}}{\mathrm{RCOP}}+\mathrm{W}_{3} \frac{\varepsilon_{\mathrm{wb}}}{R \varepsilon_{\mathrm{wb}}}+\mathrm{W}_{4} \frac{\mathrm{RA}_{\mathrm{s}}}{\mathrm{A}_{\mathrm{s}}}
$$


where $W_{i}$ represent the weights for each objective, $\mathrm{R} Q_{\text {cooling }}, \mathrm{RCOP}, R \varepsilon_{w b}$, and $R A_{s}$ are typical values of $Q_{\text {cooling }}, \mathrm{COP}$, $\varepsilon_{w b}$, and $A_{s}$,respectively.

\subsection{Particle Swarm Optimisation}

PSO is also used alongside the SMA as the second optimization algorithm to compare the results derived from both algorithms [42]. It should be mentioned that there are various versions of PSO algorithm such as basic [43] and adaptive [44] bare-bones, variable-size cooperative coevolutionary PSO [45], and hybrid optimization algorithm [46]. In this study, three different behaviours are defined for the particles in this optimization algorithm; (1) The fundamental behaviour is persistence of a particle having a random search in the solution space, (2) Tendency of the particle to redirect towards the best current solution of the whole swarm (Global best), and (3) The self-tendency of the particle to rely on its best own best solution. Eq. (10) provides the mathematical behaviour of particles over iteration $t$.

$$
\overrightarrow{P_{i}(t+1)}=\overrightarrow{P_{i}(t)}+\overrightarrow{\mathrm{V}_{i}(t+1)}
$$

where $P_{i}$ is the position of the particle $I$ and it should be bounded with upper and lower bounds based on the problem definition. The parameter "V" stands for the velocity that can cover the three explained behaviours [47] as expressed by Eq. (11).

$$
\overrightarrow{V_{i j}(t+1)}=w \cdot \overrightarrow{V_{i j}(t)}+C_{1} \cdot \overrightarrow{r_{1 i j}(t)} \cdot\left[Y_{i j}(t)-P_{i j}(t)\right]+C_{2} \cdot \overrightarrow{r_{2 i j}(t)} \cdot\left[\widehat{Y}_{i j}(t)-P_{i j}(t)\right]
$$

where $\mathrm{C}_{1}$ represents the Cognitive attraction rate, $\mathrm{C}_{2}$ represents the global attraction rate, $\hat{Y}$ stands for the global best solution/fitness and Y stands for the local best solution for each particle, $r_{1}$ and $r_{2}$ are used as additional random weights to improve the exploration and prevent trapping on local minimums. In this equation, w is the inertial coefficient that has been set to one in our implementation. Table 3 provides the initial settings for the PSO algorithm. In order to be able to compare the results derived from both SMA and PSO, the same MO fitness function, as expressed by Eq. (9), is used for both algorithms.

\section{Table 3}

(i) The table layout displayed in this section is not how it will appear in the final version. The representation below is solely purposed for providing corrections to the table. To preview the actual presentation of the table, please view the Proof.

Parameter settings for PSO.

\begin{tabular}{|l|l|}
\hline Parameter & Values \\
\hline Number of objectives & 5 \\
\hline Population number & 50 \\
\hline Max. number of iterations & 500 \\
\hline Cognitive attraction rate, c1 & 2 \\
\hline Global attraction rate, c2 & 2 \\
\hline Damping ratio of inertia coefficient & 0.99 \\
\hline Inertia coefficient & 1 \\
\hline Upper inertia & {$[3.3,0.9,3.0,0.008,200]$} \\
\hline Lower inertia & {$[2.0,0.1,0.8,0.004,100]$} \\
\hline Velocity limit & Infinite \\
\hline
\end{tabular}




\section{Future weather forecasting}

Hourly temperature and relative humidity in 2020 and 2050 are forecasted for the selected climates. Based on the operating ranges of the GIDPC, four different climates with one representative city for each climate [29] are chosen to firstly, compare the GIDPC performance in base and optimised designs, and secondly, to investigate the power saving potential of the optimized systems in both aforementioned years. Beijing with hot summer continental climate (Dwa), Doha with arid climate (BWh), Miami with tropical rainforest climate (Af), and Rome with mediterranean hot summer climate (Csa) are the chosen cities.

\subsection{Weather data creation}

The Intergovernmental Panel for Climate Change (IPCC) developed a quantity of probable scenarios of future greenhouse gas emissions based on the socio-economic data and scenarios to project the future climate variations for impact and adaptation assessment [48]. A number of emissions pathways are presented through the Special Report on Emission Scenarios (SRES) and then through the sets of emission scenarios called Representative Concentration Pathways (RCPs).

In this paper, IPCC's SRES A2 climate scenario that represents a high emission future scenario is considered in order to calculate the operating hours for GIDPC in both 2020 and 2050. It is worth mentioning that in two sets of scenarios (SRES and RCPs), two high emission scenarios, i.e., A2 and RCP8.5, are equivalent [49,50]. Meteonorm software [51] is used to generate the hourly temperature and relative humidity data for the selected cities. In this study, based on the operating ranges of the GIDPC, the temperatures above $25^{\circ} \mathrm{C}$ are selected as the operating conditions in order to calculate the operating hours.

\subsection{Hourly data}

Fig. 5 shows the number of operating hours for all climates in 2020 and 2050. According to the results, in all climates, the number of operating hours are increased by 2050 as a result of global warming. In hot summer continental and Mediterranean hot summer, the GIDPC is not needed in winter (December-February) while in other two climates, it is needed to operate in all seasons.

\section{Fig. 5}

(a)

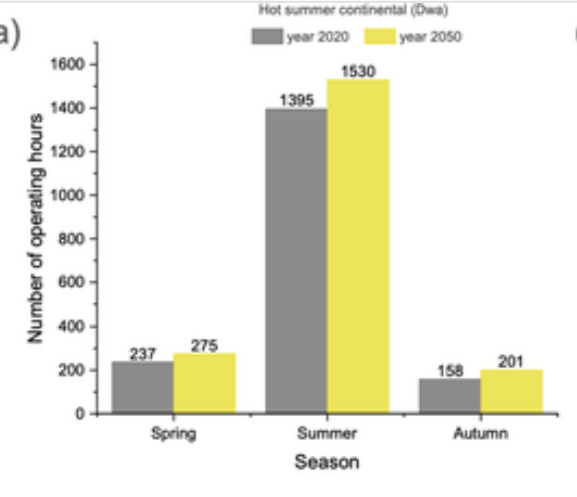

(c)

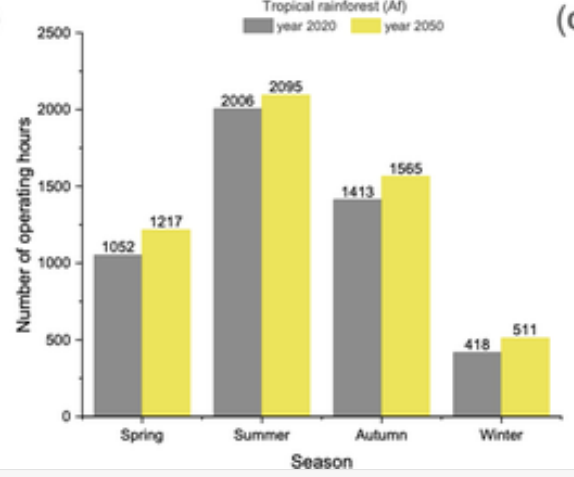

(b)

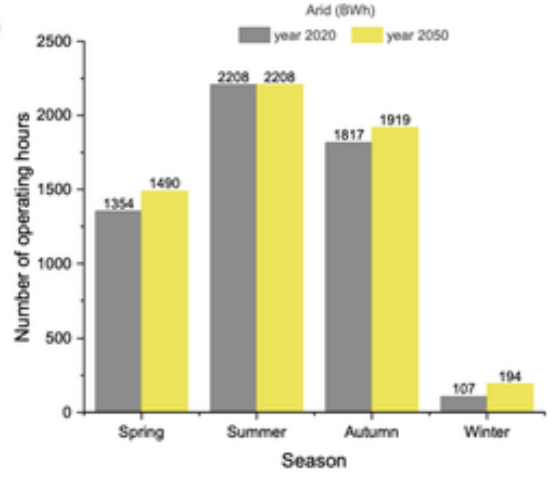

(d)

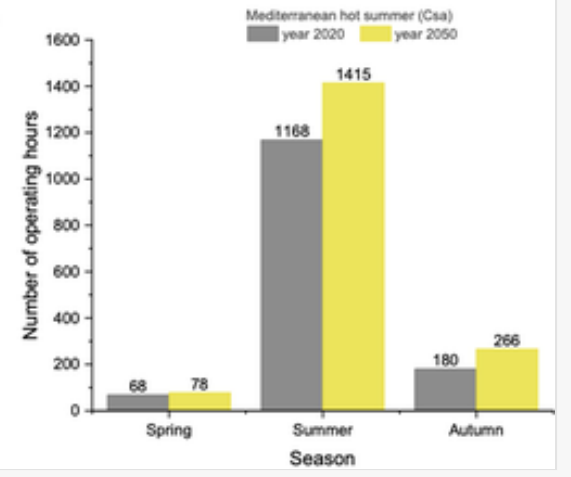




\subsection{Optimization results}

The optimum value of the decision variables in each climate is identified by both SMA and PSO. The results are derived based on the average value of the air properties, i.e., temperature and relative humidity, over the operating hours in 2020. This is done because a single optimized unit in each climate is aimed to operate for the next 30 years. Five scenarios are considered for each climate in which different weight distributions are assumed in order to assess and compare different weight distributions over the objectives. The primary scenario is to equally distribute the weights over selected objectives (multi objective). It means that importance of objectives for the optimization algorithms is same while in other four scenarios weight distribution is focused on one objective (single objective). This means that optimization algorithm will mainly focus on maximising/minimising that particular objective. This allows us to observe and compare performance of the system under each scenario.

The comprehensive results derived from PSO and SMA are listed in Table 4 and Table 5 respectively. In addition, the base system properties are listed in the above tables [27]. The results revealed that identified optimum decision variables by PSO and SMA are similar. In addition, compared to the base system, the equally distributed weights lead to better results in terms of cooling capacity and COP while other objectives, i.e., wet bulb efficiency and surface area, remain almost same. However, the system performance under other single objective scenarios is not satisfying. Although, the third scenario where COP is considered as the dominant objective (W3 $=0.85$ ) leads to convincing results, where COP is maximised and the surface area is lower than the base system, but cooling capacity which is one of the main performance parameters are lower than the base system. This trend is same in scenario 5 where surface area is the dominant objective (W4 $=0.85$ ). High surface area in second scenario where cooling capacity is the dominant objective (W1 $=0.85)$ and low cooling capacity in fourth scenario where wet bulb efficiency is the dominant objective (W3 $=0.85$ ) have made these scenarios unsatisfactory. As a consequence, scenario 1 in both PSO and SMA methods is selected to discuss optimization results and to investigate performance of the optimized systems.

\section{Table 4}

(i) The table layout displayed in this section is not how it will appear in the final version. The representation below is solely purposed for providing corrections to the table. To preview the actual presentation of the table, please view the Proof.

Optimisation results by PSO.

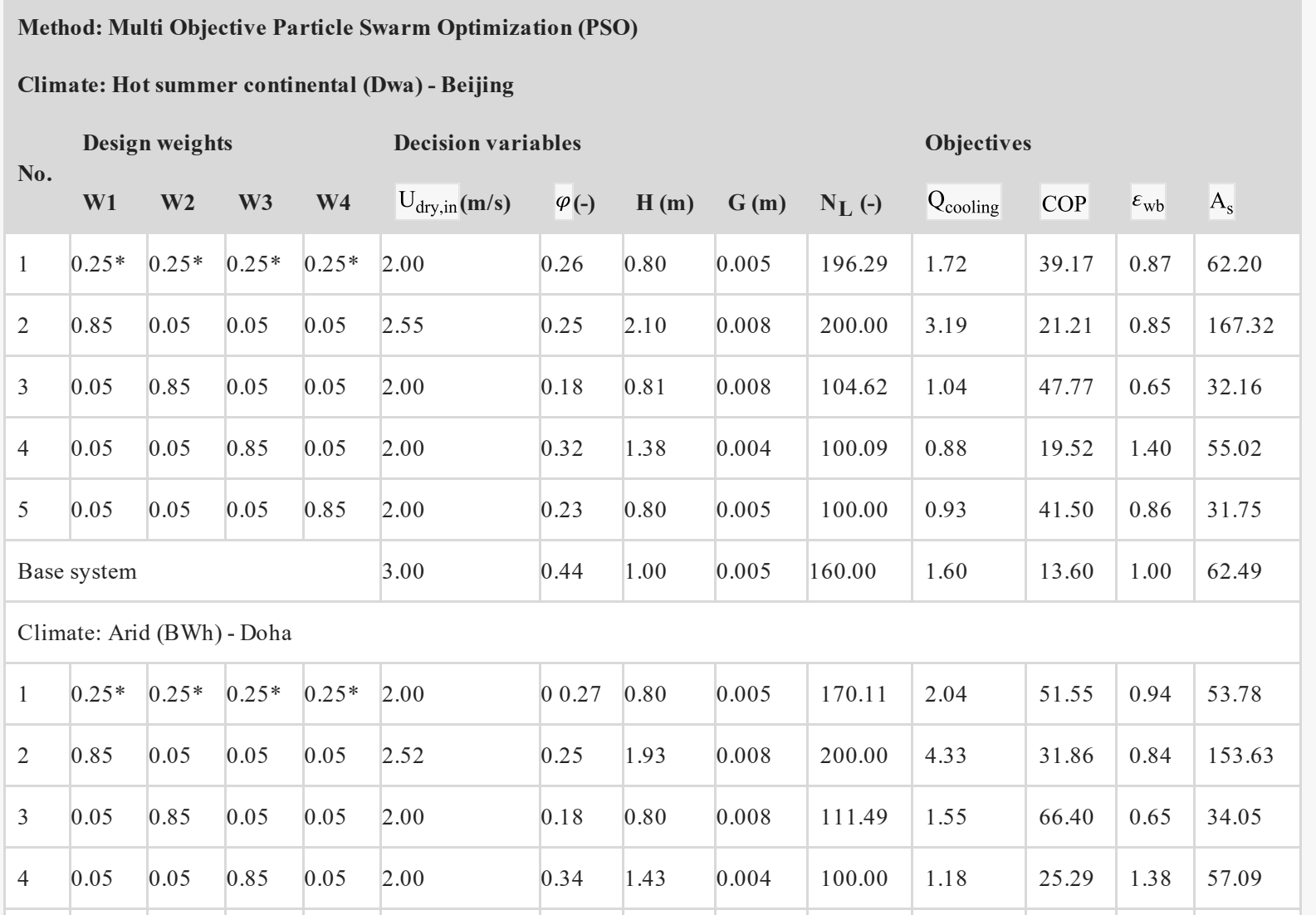




\begin{tabular}{|c|c|c|c|c|c|c|c|c|c|c|c|c|c|}
\hline 5 & 0.05 & 0.05 & 0.05 & 0.85 & 2.00 & 0.24 & 0.80 & 0.005 & 100.00 & 1.26 & 53.86 & 0.92 & 31.75 \\
\hline & ste & & & & 3.0 & 0.44 & 1.00 & 0.005 & 160.00 & 2.22 & 18.88 & 1.00 & 62.49 \\
\hline
\end{tabular}

Climate: Tropical rainforest (Af) - Miami

\begin{tabular}{|l|l|l|l|l|l|l|l|l|l|l|l|l|l|}
\hline 1 & $0.25^{*}$ & $0.25^{*}$ & $0.25^{*}$ & $0.25^{*}$ & 2.00 & 0.24 & 0.80 & 0.006 & 200.00 & 1.39 & 31.96 & 0.83 & 63.38 \\
\hline 2 & 0.85 & 0.05 & 0.05 & 0.05 & 2.53 & 0.24 & 2.20 & 0.008 & 200.00 & 2.48 & 16.12 & 0.87 & 175.00 \\
\hline 3 & 0.05 & 0.85 & 0.05 & 0.05 & 2.00 & 0.17 & 0.80 & 0.008 & 100.00 & 0.79 & 38.01 & 0.67 & 31.75 \\
\hline 4 & 0.05 & 0.05 & 0.85 & 0.05 & 2.00 & 0.30 & 1.31 & 0.004 & 100.00 & 0.71 & 16.84 & 1.44 & 51.84 \\
\hline 5 & 0.05 & 0.05 & 0.05 & 0.85 & 2.00 & 0.21 & 0.80 & 0.006 & 100.00 & 0.75 & 34.35 & 0.85 & 31.75 \\
\hline Base system & & & 3.00 & 0.44 & 1.00 & 0.005 & 160.00 & 1.21 & 10.25 & 1.01 & 62.49 \\
\hline
\end{tabular}

Climate: Mediterranean hot summer (Csa) - Rome

\begin{tabular}{|l|l|l|l|l|l|l|l|l|l|l|l|l|l|}
\hline 1 & $0.25^{*}$ & $0.25^{*}$ & $0.25^{*}$ & $0.25^{*}$ & 2.00 & 0.27 & 0.80 & 0.005 & 199.43 & 1.83 & 40.78 & 0.88 & 63.21 \\
\hline 2 & 0.85 & 0.05 & 0.05 & 0.05 & 2.51 & 0.26 & 2.09 & 0.008 & 200 & 3.35 & 23.08 & 0.85 & 165.91 \\
\hline 3 & 0.05 & 0.85 & 0.05 & 0.05 & 2.00 & 0.18 & 0.80 & 0.008 & 107.69 & 1.12 & 50.05 & 0.64 & 32.80 \\
\hline 4 & 0.05 & 0.05 & 0.85 & 0.05 & 2.00 & 0.35 & 1.40 & 0.004 & 100.00 & 0.91 & 19.82 & 1.39 & 55.81 \\
\hline 5 & 0.05 & 0.05 & 0.05 & 0.85 & 2.00 & 0.24 & 0.80 & 0.005 & 100.00 & 0.98 & 43.12 & 0.85 & 31.75 \\
\hline Base system & & & 3.00 & 0.44 & 1.00 & 0.005 & 160.00 & 1.71 & 14.52 & 0.99 & 62.49 \\
\hline
\end{tabular}

\section{Table 5}

(i) The table layout displayed in this section is not how it will appear in the final version. The representation below is solely purposed for providing corrections to the table. To preview the actual presentation of the table, please view the Proof.

Optimisation results by SMA.

\begin{tabular}{|c|c|c|c|c|c|c|c|c|c|c|c|c|c|}
\hline \multicolumn{14}{|c|}{ Method: Slime Mould Algorithm (SMA) } \\
\hline \multicolumn{14}{|c|}{ Climate: Hot summer continental (Dwa) - Beijing } \\
\hline \multirow{2}{*}{ No. } & \multicolumn{4}{|c|}{ Design weights } & \multicolumn{5}{|c|}{ Decision variables } & \multicolumn{4}{|c|}{ Objectives } \\
\hline & W1 & W2 & W3 & W4 & $\mathrm{U}_{\mathrm{dry}, \text { in }}(\mathbf{m} / \mathbf{s})$ & $\varphi(-)$ & $\mathbf{H}(\mathbf{m})$ & G (m) & $\mathbf{N}_{\mathbf{L}}(-)$ & $\mathrm{Q}_{\text {cooling }}$ & $\mathrm{COP}$ & $\varepsilon_{\mathrm{wb}}$ & $\mathrm{A}_{\mathrm{s}}$ \\
\hline 1 & $0.25^{*}$ & $0.25 *$ & $0.25 *$ & $0.25 *$ & 2.00 & 0.26 & 0.80 & 0.004 & 128.03 & 1.06 & 33.12 & 1.07 & 40.70 \\
\hline 2 & 0.85 & 0.05 & 0.05 & 0.05 & 2.30 & 0.24 & 1.60 & 0.008 & 200.00 & 2.72 & 28.35 & 0.81 & 126.79 \\
\hline 3 & 0.05 & 0.85 & 0.05 & 0.05 & 2.00 & 0.18 & 0.81 & 0.008 & 103.34 & 1.03 & 47.79 & 0.65 & 31.99 \\
\hline 4 & 0.05 & 0.05 & 0.85 & 0.05 & 2.00 & 0.32 & 1.41 & 0.004 & 100.00 & 0.88 & 19.30 & 1.40 & 56.28 \\
\hline 5 & 0.05 & 0.05 & 0.05 & 0.85 & 2.00 & 0.22 & 0.80 & 0.005 & 100.00 & 0.93 & 41.23 & 0.86 & 31.75 \\
\hline \multicolumn{5}{|c|}{ Base system } & 3.00 & 0.44 & 1.00 & 0.005 & 160.00 & 1.60 & 13.60 & 1.00 & 62.49 \\
\hline \multicolumn{14}{|c|}{ Climate: Arid (BWh) - Doha } \\
\hline 1 & $0.25^{*}$ & $0.25^{*}$ & $0.25 *$ & $0.25 *$ & 2.00 & 0.22 & 0.80 & 0.004 & 141.36 & 1.65 & 48.54 & 0.98 & 44.99 \\
\hline 2 & 0.85 & 0.05 & 0.05 & 0.05 & 3.02 & 0.22 & 1.40 & 0.008 & 200.00 & 4.26 & 27.27 & 0.67 & 111.59 \\
\hline 3 & 0.05 & 0.85 & 0.05 & 0.05 & 2.00 & 0.18 & 0.80 & 0.008 & 110.21 & 1.53 & 66.38 & 0.65 & 33.58 \\
\hline 4 & 0.05 & 0.05 & 0.85 & 0.05 & 2.00 & 0.34 & 1.41 & 0.004 & 100.00 & 1.18 & 25.56 & 1.38 & 56.25 \\
\hline 5 & 0.05 & 0.05 & 0.05 & 0.85 & 2.00 & 0.24 & 0.80 & 0.004 & 100.00 & 1.16 & 44.82 & 1.08 & 31.75 \\
\hline \multicolumn{5}{|c|}{ Base system } & 3.00 & 0.44 & 1.00 & 0.005 & 160.00 & 2.22 & 18.88 & 1.00 & 62.49 \\
\hline
\end{tabular}


Climate: Tropical rainforest (Af) - Miami

\begin{tabular}{|l|l|l|l|l|l|l|l|l|l|l|l|l|l|}
\hline 1 & $0.25^{*}$ & $0.25^{*}$ & $0.25^{*}$ & $0.25^{*}$ & 2.00 & 0.24 & 0.80 & 0.006 & 199.86 & 1.40 & 32.83 & 0.79 & 63.33 \\
\hline 2 & 0.85 & 0.05 & 0.05 & 0.05 & 2.28 & 0.23 & 2.22 & 0.008 & 200.00 & 2.33 & 18.93 & 0.90 & 176.89 \\
\hline 3 & 0.05 & 0.85 & 0.05 & 0.05 & 2.00 & 0.16 & 0.80 & 0.008 & 100.00 & 0.79 & 38.04 & 0.67 & 31.75 \\
\hline 4 & 0.05 & 0.05 & 0.85 & 0.05 & 2.00 & 0.29 & 1.32 & 0.004 & 100.00 & 0.71 & 16.80 & 1.44 & 52.27 \\
\hline 5 & 0.05 & 0.05 & 0.05 & 0.85 & 2.00 & 0.21 & 0.80 & 0.005 & 100.96 & 0.75 & 33.49 & 0.88 & 31.79 \\
\hline Base system & & & 3.00 & 0.44 & 1.00 & 0.005 & 160.00 & 1.21 & 10.25 & 1.01 & 62.49 \\
\hline
\end{tabular}

Climate: Mediterranean hot summer (Csa) - Rome

\begin{tabular}{|l|l|l|l|l|l|l|l|l|l|l|l|l|l|l|}
\hline 1 & $0.25^{*}$ & $0.25^{*}$ & $0.25^{*}$ & $0.25^{*}$ & 2.00 & 0.27 & 0.82 & 0.006 & 158.41 & 1.52 & 42.34 & 0.84 & 51.19 \\
\hline 2 & 0.85 & 0.05 & 0.05 & 0.05 & 2.23 & 0.27 & 1.89 & 0.008 & 200 & 3.00 & 28.84 & 0.88 & 150.16 \\
\hline 3 & 0.05 & 0.85 & 0.05 & 0.05 & 2.00 & 0.18 & 0.80 & 0.008 & 104.96 & 1.10 & 50.03 & 0.65 & 32.21 \\
\hline 4 & 0.05 & 0.05 & 0.85 & 0.05 & 2.00 & 0.34 & 1.39 & 0.004 & 100.00 & 0.91 & 19.90 & 1.39 & 55.41 \\
\hline 5 & 0.05 & 0.05 & 0.05 & 0.85 & 2.00 & 0.21 & 0.80 & 0.006 & 100.00 & 1.01 & 46.57 & 0.76 & 31.75 \\
\hline Base system & & & 3.00 & 0.44 & 1.00 & 0.005 & 160.00 & 1.71 & 14.52 & 0.99 & 62.49 \\
\hline
\end{tabular}

\subsection{Decision variables}

In all climates optimum velocity of the intake air derived by both PSO and SMA is $2(\mathrm{~m} / \mathrm{s})$ which is lower than the velocity in base system, i.e., $3(\mathrm{~m} / \mathrm{s})$. This is resulted from a robust trade-off by the optimization algorithms as higher velocity leads to higher pressure drop and to lower COP values which is not preferred [27] but could lead to more heat and mass transfer rate within the HMX. This low value was expected as the higher COP was aimed.

The optimum working air ratio by both PSO and SMA methods in all four climates are in the range of $0.22--0.27$ which are lower than the working air ratio in base system, i.e., 0.44 . The working air ratio is proportion of the exhaust air to intake air which its higher value will lead to more temperature drop, more efficiencies but will bring down the COP and cooling capacity. The identified working air ratio by both methods in hot summer, typical rainforest and Mediterranean hot summer climates are same which are $0.26,0.24$ and 0.27 respectively. But the identified working air ratio in Arid climate identified by PSO is 0.27 and by SMA is 0.22 .

The optimum HMX height identified by PSO and SMA is in narrow range of 0.80-0.82 (m) which is lower than the base system height, i.e., 1 (m). A GIDPC with longer HMX has better performance in terms of cooling capacity but it brings up negative effects by increasing the surface area, pressure drop and fan power consumption [10]. Therefore, although it provides the users with more efficient system but it can't be an economical choice. As a consequence, over a trade-off, it is concluded by both optimization methods that the optimum HMX height is $0.8(\mathrm{~m})$ in all climates.

The channel gap in the base system is $0.005(\mathrm{~m})$ while it is revealed that the optimum values are different. Base on the PSO method, the optimum channel gap in tropical rainforest climate is $0.006(\mathrm{~m})$ while it is same as the base system in other climates. However, based on SMA results, it holds the optimum value of $0.004(\mathrm{~m})$ in hot summer continental and arid climates, and $0.006(\mathrm{~m})$ in tropical rainforest and Mediterranean hot summer climates. High pressure drop can be recorded in smaller channel gap values which requires higher fan power consumption while larger channel gap can lead to better cooling capacity values.

Higher number of layers will bring about more power consumption as a result of higher pressure drop and adds up to the construction and running cost. However, it can improve the system performance in terms of cooling capacity. The number of layers in the base system is 160 while the optimum numbers in PSO ranges from 170.11 to 200.00 and in SMA it is in the range of 128.03-199.86.

\subsection{Performance of the optimized system in 2020 and 2050}

In this section the hourly COP of base system is compared with the optimized systems by PSO and SMA in all climates in years 2020 and 2050. In addition, the average value of the cooling capacity, COP and wet bulb efficiency in 
operating hours are compared for all systems. The performance of the base system is calculated based on the base system properties which were listed in Tables 4 and 5, i.e., air velocity of $3(\mathrm{~m} / \mathrm{s})$, working air ratio of 0.44 , HMX height of 1(m), Gap of $0.005(\mathrm{~m})$ and number of layers of 160. Furthermore, the performance of the SMA and PSO systems are calculated using the selected optimum decision variables from the previous section. In general, the COP of the PSO and SMA systems are superior than the base system while the identified decision variables by SMA and PSO play the key role in introducing the superior optimisation methods. The differences between the SMA and PSO are the results of the robust trade-offs made by the optimisation methods by considering the advantageous and disadvantageous of the identified optimum decision values.

Fig. 6 shows the hourly COP of three systems over the operating hours in each climate with an enlarged view for period of hours for better visualization. As shown in Fig. 6 (a), the COP of the systems in hot summer continental climate is demonstrated over the operating hours. As expected, the optimised system outperforms the base system. However, COP in PSO outperforms the SMA in both years which is mainly due to the differences in gap, i.e., 0.005 in PSO versus 0.004 in SMA, and number of layers, i.e., 196.29 in PSO versus 128.03 in SMA.
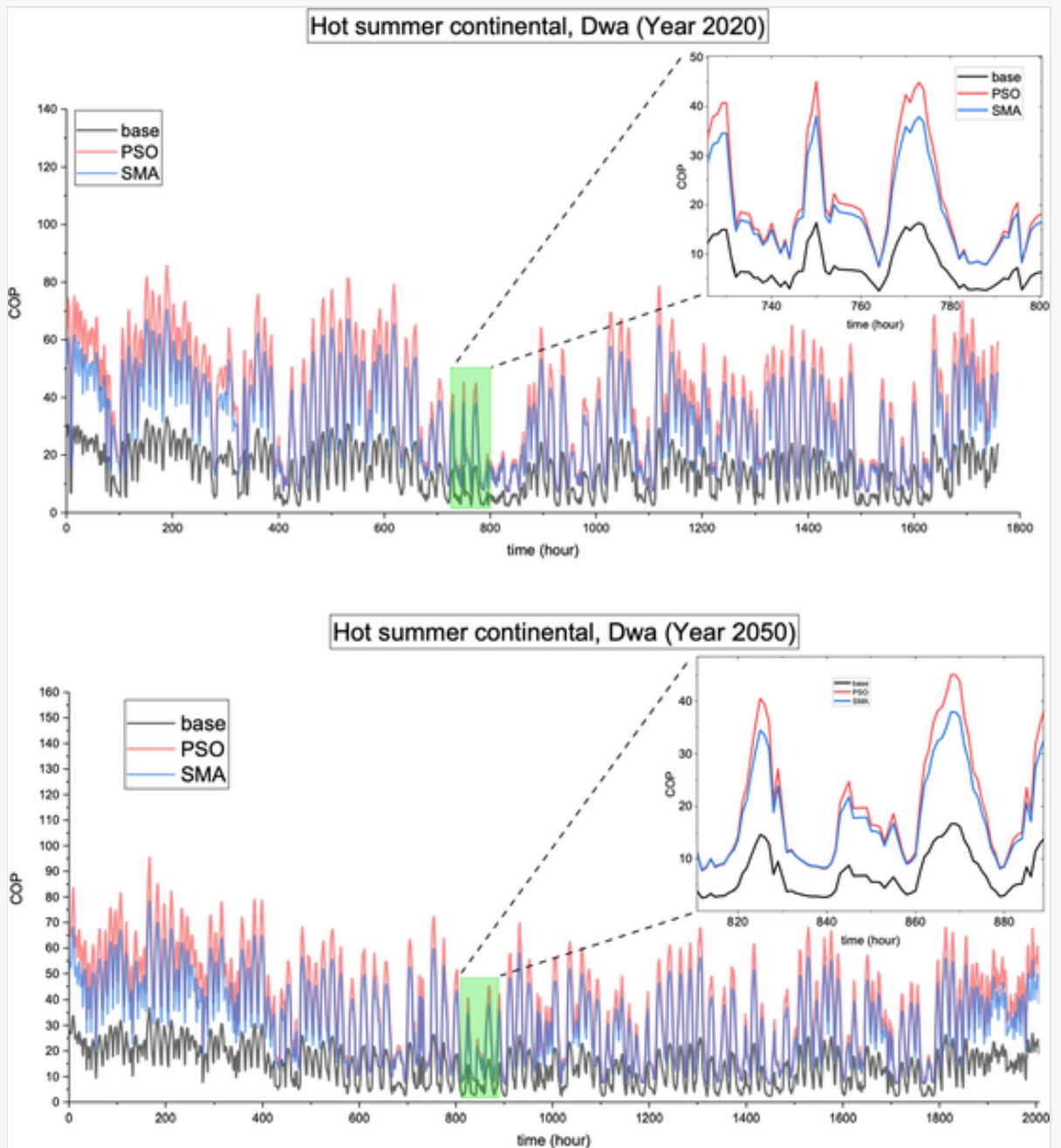

(a) 


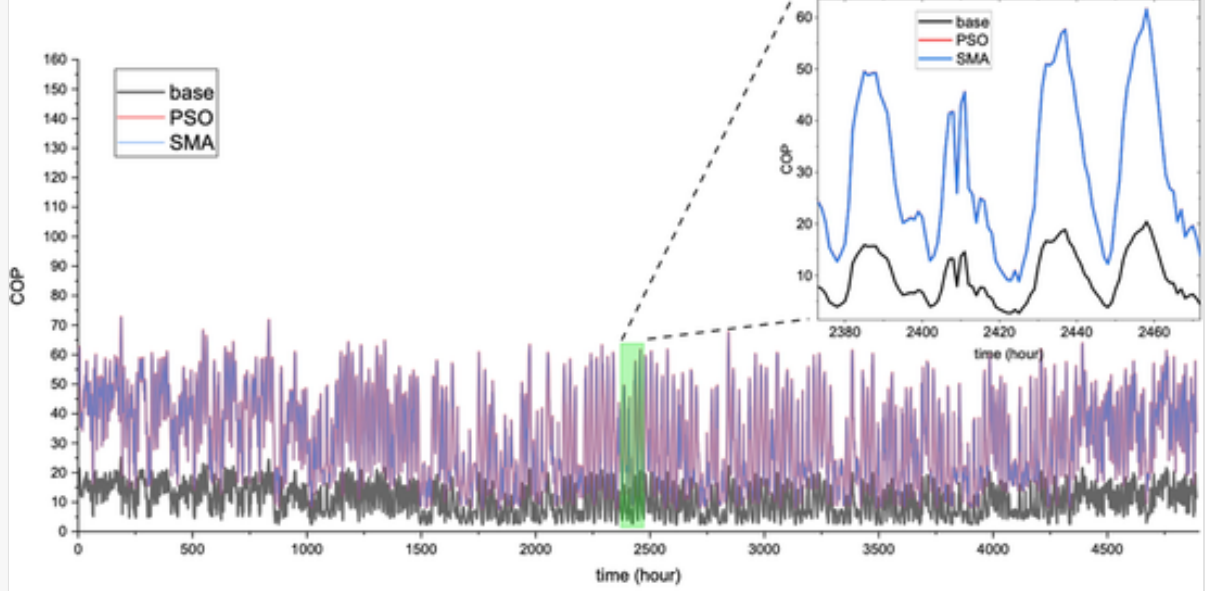

Tropical rainforest, Af (Year 2050)

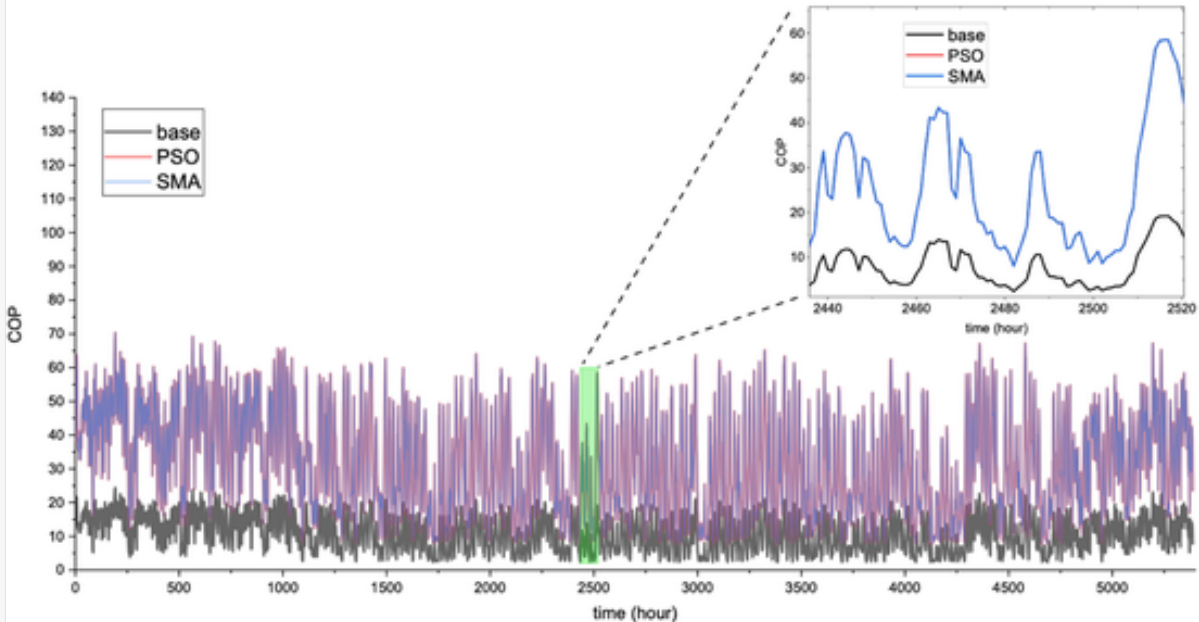

(c) 

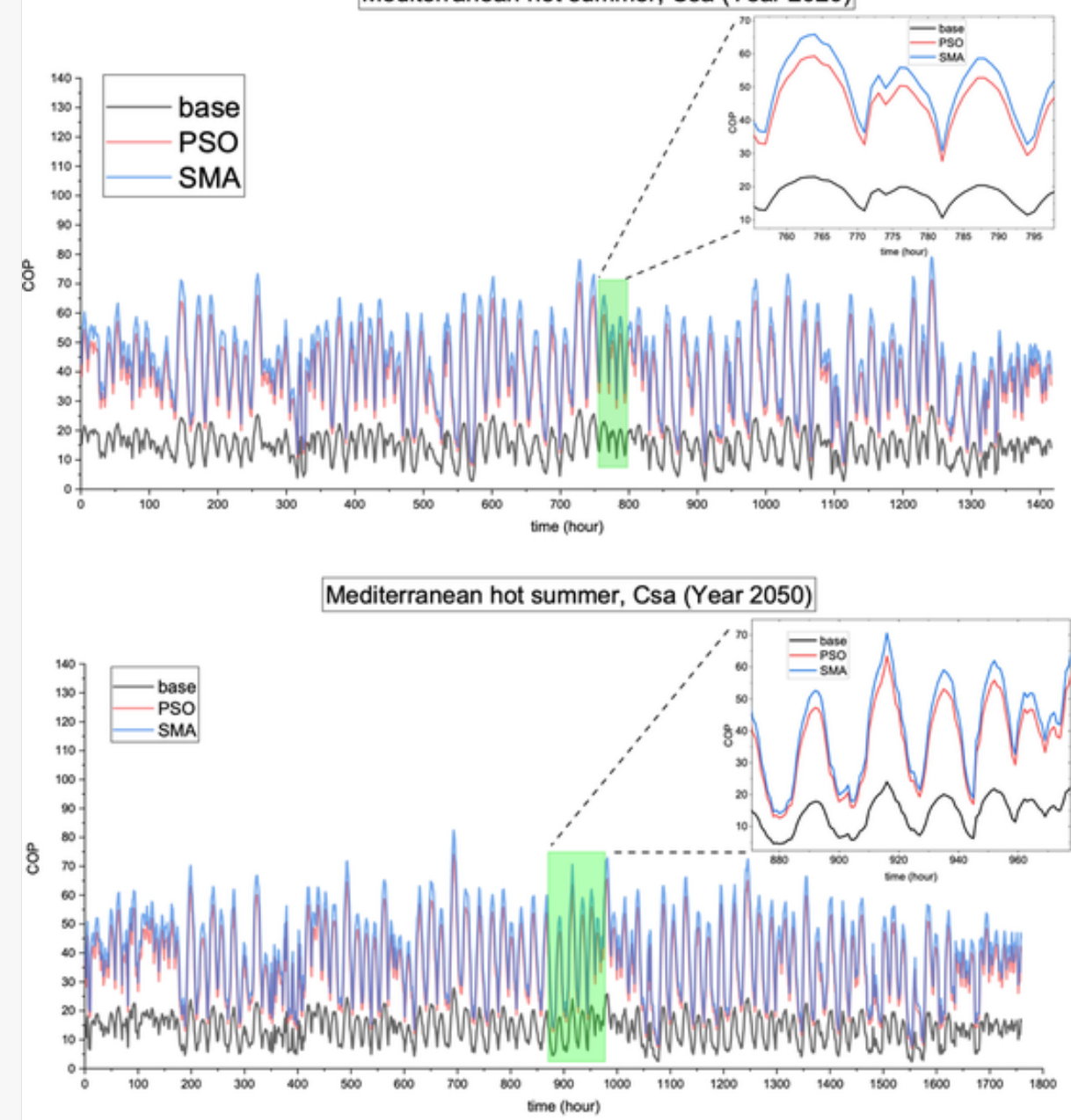

(d)

In 2020, the COP of the base system is in the range of 2.28-33.16 while the COP of the improved system by PSO ranges from 7.14 to 85.53 , and by SMA is in the range of 7.22-70.39. The trend is similar in 2050 where the COP of the base system varies from 2.32 to 36.87 while in PSO it ranges from 6.89 to 95.46 and in SMA it is in the range of 7.05-78.26.

As seen in Fig. 6 (b), the system behaviour in Arid climate is similar to the hot summer continental climate where the PSO outperforms the SMA and base system in both years. This is because of the higher working air ratio, larger gap and a greater number of layers in PSO which have led to slightly better COP values. In 2020, the COP of the base system is in the range of 2.39-43.74. This range is significantly improved when the optimized systems are in operation. The COP ranges from 7.37 to 116.78 in PSO system and it is in the range of 7.95-100.61 in SMA system. This trend 
continues in 2050 when the COP of the base system is in the range of $2.47-45.72$ while in SMA it is in the range of 8.45-105.37 and the performance is occurred in PSO system where the COP varies from 7.73 to 122.38.

As seen in Fig. 6 (c), the overall COP values of the optimized systems in tropical rainforest climate are improved compared with the base system in both years. However, owing to the identical optimum parameters derived by both PSO and SMA methods, the performance of both systems are almost same. In 2020, the COP of the base system is in the range of 2.31-25.13 while in PSO and SMA systems, it is in the same range of 7.61-72.73. The similar behaviour in both optimized systems were expected due to the extremely similar identified optimum operating and design parameters. In 2050, the COP of the base system varies from 2.31 to 24.37 while in the systems optimized by PSO and SMA, it is in the same range of 7.58-70.37.

In Mediterranean hot summer the optimized systems outperform the base system in terms of COP where the SMA has slightly performs better than PSO in both 2020 and 2050. This is because of the differences in optimum values of three parameters, i.e., HMX height, gap and number of layers by both methods. As seen in Fig. 6 (d), in 2020, the COP of the base system was in the range of 2.83-28.37 while it is improved to the ranges of 8.10-71.23 and 9-09-79.01 by PSO and SMA respectively. Same behaviour is occurred in 2050 when the COP of the base system was in the range of 2.39-27.85 while the SMA and PSO have increased the ranges to 7.97-82.41 and 7.06-73.94 respectively. This superior performance by SMA is mainly due to the slightly higher HMX height compared with the SMA.

Three Empirical Cumulative Distribution Function (ECDF)-based statistical distance measures, i.e., Kolmogorov Smirnov Test, Kuiper Test, and Anderson Darling Test, are selected to analyse the results. We have focused on COP estimation in the four climates in 2020 and 2050. The Table 6 shows the statistical analysis of the PSO and SMA results in comparison to the base model. It should be noted that all measures have valid values with the p-value less than 0.01. As can be seen in the Table 6, from a statistical point of view, in tropical rainforest 2020 and 2050 both PSO and SMA have the same statistical distance from the base model. While in hot summer continental and Arid climates, PSO results have a higher rate of statistical differences and in Mediterranean hot summer the SMA results have a higher rate of statistical differences.

\section{Table 6}

(i) The table layout displayed in this section is not how it will appear in the final version. The representation below is solely purposed for providing corrections to the table. To preview the actual presentation of the table, please view the Proof.

Statistical analysis of the PSO and SMA results.

\begin{tabular}{|c|c|c|c|}
\hline \multirow{2}{*}{ Test name } & PSO vs. Base & SMA vs. Base & PSO vs. SMA \\
\hline & \multicolumn{3}{|c|}{ Hot summer continental (Dwa) - Beijing 2020 - COP Estimation } \\
\hline Kolmogorov Smirnov Test & 0.4730 & 0.4217 & 0.1239 \\
\hline Kuiper Test & 0.4730 & 0.4217 & 0.1282 \\
\hline \multirow[t]{2}{*}{ Anderson Darling Test } & 0.3187 & 0.2766 & 0.0313 \\
\hline & \multicolumn{3}{|c|}{ Hot summer continental (Dwa) - Beijing 2050 - COP Estimation } \\
\hline Kolmogorov Smirnov Test & 0.5434 & 0.4933 & 0.1472 \\
\hline Kuiper Test & 0.5434 & 0.4933 & 0.1519 \\
\hline \multirow[t]{2}{*}{ Anderson Darling Test } & 0.4385 & 0.3813 & 0.0403 \\
\hline & \multicolumn{3}{|c|}{ Arid (BWh) - Doha 2020 - COP Estimation } \\
\hline Kolmogorov Smirnov Test & 0.8399 & 0.7290 & 0.1705 \\
\hline Kuiper Test & 0.8399 & 0.7290 & 0.1705 \\
\hline \multirow[t]{2}{*}{ Anderson Darling Test } & 1.1154 & 0.9861 & 0.0763 \\
\hline & \multicolumn{3}{|c|}{ Arid $(\mathrm{BWh})$ - Doha 2050 - COP Estimation } \\
\hline
\end{tabular}




\begin{tabular}{|c|c|c|c|}
\hline Kolmogorov Smirnov Test & 0.8136 & 0.7147 & 0.1662 \\
\hline Kuiper Test & 0.8136 & 0.7147 & 0.1662 \\
\hline \multirow[t]{2}{*}{ Anderson Darling Test } & 1.0827 & 0.9489 & 0.0670 \\
\hline & \multicolumn{3}{|c|}{ Tropical rainforest (Af) - Miami 2020 - COP Estimation } \\
\hline Kolmogorov Smirnov Test & 0.7622 & 0.7622 & 0.0035 \\
\hline Kuiper Test & 0.7622 & 0.7622 & 0.0052 \\
\hline \multirow[t]{2}{*}{ Anderson Darling Test } & 0.7994 & 0.7994 & 0.0000 \\
\hline & \multicolumn{3}{|c|}{ Tropical rainforest (Af) - Miami 2050 - COP Estimation } \\
\hline Kolmogorov Smirnov Test & 0.7756 & 0.7756 & 0.0026 \\
\hline Kuiper Test & 0.7756 & 0.7756 & 0.0052 \\
\hline \multirow[t]{2}{*}{ Anderson Darling Test } & 0.8240 & 0.8241 & 0.0000 \\
\hline & \multicolumn{3}{|c|}{ Mediterranean hot summer (Csa) - 2020 - COP Estimation } \\
\hline Kolmogorov Smirnov Test & 0.5145 & 0.5378 & 0.0889 \\
\hline Kuiper Test & 0.5145 & 0.5378 & 0.0889 \\
\hline \multirow[t]{2}{*}{ Anderson Darling Test } & 0.2819 & 0.2974 & 0.0131 \\
\hline & \multicolumn{3}{|c|}{ Mediterranean hot summer (Csa) - 2050 - COP Estimation } \\
\hline Kolmogorov Smirnov Test & 0.6029 & 0.6379 & 0.1032 \\
\hline Kuiper Test & 0.6029 & 0.6379 & 0.1032 \\
\hline Anderson Darling Test & 0.4281 & 0.4628 & 0.0181 \\
\hline
\end{tabular}

In order to further compare the impact of optimization algorithms on the system performance, the average value of three objectives, i.e., cooling capacity, COP and wet bulb efficiency, over the operating hours are summarized in Table 7. In terms of COP, the performance of the optimized systems has significantly improved by both PSO and SMA methods. However, in terms of cooling capacity and wet bulb efficiency, the changes are not same.

\section{Table 7}

(i) The table layout displayed in this section is not how it will appear in the final version. The representation below is solely purposed for providing corrections to the table. To preview the actual presentation of the table, please view the Proof.

Average value of three performance parameters in base and optimized systems.

\begin{tabular}{|c|c|c|c|c|c|c|c|c|c|c|c|c|c|}
\hline \multirow{2}{*}{ Year } & \multirow{2}{*}{$\begin{array}{l}\text { Climate } \\
\text { Objective }\end{array}$} & \multicolumn{3}{|c|}{$\begin{array}{l}\text { Hot summer continental } \\
\text { (Dwa) }\end{array}$} & \multicolumn{3}{|c|}{ Arid (BWh) } & \multicolumn{3}{|c|}{$\begin{array}{l}\text { Tropical rainforest } \\
\text { (Af) }\end{array}$} & \multicolumn{3}{|c|}{$\begin{array}{l}\text { Mediterranean hot summer } \\
\text { (Csa) }\end{array}$} \\
\hline & & Base & PSO & SMA & Base & PSO & SMA & Base & PSO & SMA & Base & PSO & SMA \\
\hline \multirow{3}{*}{2020} & $\frac{\mathrm{Q}_{\text {cooling }}}{(\mathrm{kW})}$ & 1.68 & 1.71 & 1.06 & 2.31 & 2.08 & 1.61 & 1.26 & 1.41 & 1.41 & 1.74 & 1.78 & 1.54 \\
\hline & $\mathrm{COP}$ & 14.27 & 37.56 & 31.69 & 19.57 & 51.78 & 44.92 & 10.73 & 32.70 & 32.70 & 14.80 & 38.55 & 42.86 \\
\hline & $\varepsilon_{\mathrm{wb}}$ & 1.01 & 0.98 & 1.23 & 1.00 & 0.97 & 1.07 & 1.02 & 0.86 & 0.86 & 0.99 & 0.95 & 0.85 \\
\hline \multirow{3}{*}{2050} & $\begin{array}{l}\mathrm{Q}_{\text {cooling }} \\
(\mathrm{kW})\end{array}$ & 1.67 & 1.72 & 1.06 & 2.32 & 2.10 & 1.64 & 1.26 & 1.42 & 1.41 & 1.60 & 1.65 & 1.42 \\
\hline & $\mathrm{COP}$ & 14.21 & 37.51 & 31.67 & 19.66 & 52.30 & 45.52 & 10.68 & 32.71 & 32.71 & 13.61 & 35.71 & 39.73 \\
\hline & $\varepsilon_{\mathrm{wb}}$ & 1.01 & 0.98 & 1.23 & 1.00 & 0.98 & 1.08 & 1.03 & 0.86 & 0.87 & 1.00 & 0.97 & 0.87 \\
\hline
\end{tabular}


In 2020, the cooling capacity of the PSO system in hot summer continental, tropical rainforest and Mediterranean is better than the base system while it is almost unchanged in Arid climate. However, for the optimized system by SMA, except in tropical rainforest where the cooling capacity is better than the base system, in other climates the cooling capacity is lower than the base system. Although the operating hours in 2050 has increased in all climates but the behaviour of the systems remain exactly the same as 2020 .

In terms of wet bulb efficiency, the performance of the systems follows the same trend in both 2020 and 2050. In hot summer continental, arid and Mediterranean climates, the wet bulb efficiency of the optimized system by PSO is slightly decreased (almost unchanged) but in tropical rainforest it is decreased by $16 \%$ by reaching the average value of 0.86. The performance of the SMA system, compared with the base system, outperforms in hot summer continental and Arid climates while it holds the lower average values in tropical rainforest and Mediterranean hot summer climates.

As listed in Tables 4 and 5, the surface area of the base system with 200 layers is $62.49\left(\mathrm{~m}^{2}\right)$ whereas the optimized systems have lower values in some climates. For instance, in the selected scenario (No.1), for SMA system, except tropical rainforest climate in which the surface area is same as the base system, in all other climates, owing to a smaller number of layers, it has decreased. However, in the PSO system, the surface area is only decreased in Arid climate, i.e., $53.78\left(\mathrm{~m}^{2}\right)$ while in all other climates the it is almost unchanged.

\subsection{Energy saving potential of the optimized systems}

The power consumption of the GIDPC considering the forecasted operating hours in 2020 and 2050 is analysed to assess the energy saving potential of the optimized systems. The power consumption values are calculated for the three existed systems, i.e., base, optimized by PSO and optimized by SMA. The basis of the calculations is the rate of power consumption for each system which is calculated considering the system performance in terms of cooling capacity and COP. The rate of power consumption for the base system is around $117.5 \mathrm{~W}$ while it is around $45 \mathrm{~W}$ for the PSO system and around $33 \mathrm{~W}$ for SMA system. The values are in line with the presented cooling capacity and COP values for each of three systems.

Based on the operating hours in each climate, the total power consumption is calculated in both 2020 and 2050. As seen in Fig. 7 (a), in the hot summer continental climate in 2020, the power consumption is $211.00(\mathrm{kWh})$ for the base system whereas it has reduced to $80.55(\mathrm{kWh})$ and $59.07(\mathrm{kWh})$ for PSO and SMA respectively. In spite of higher operating hours in 2050, the trend is same and the optimized systems perform economically. The power consumption of the base system is $236.46(\mathrm{kWh})$ whereas it is $90.27(\mathrm{kWh})$ for the PSO and $66.19(\mathrm{kWh})$ the SMA systems. This means that in both years the power savings of $61.82 \%$ and $72.00 \%$ can occur by the PSO and SMA respectively. 

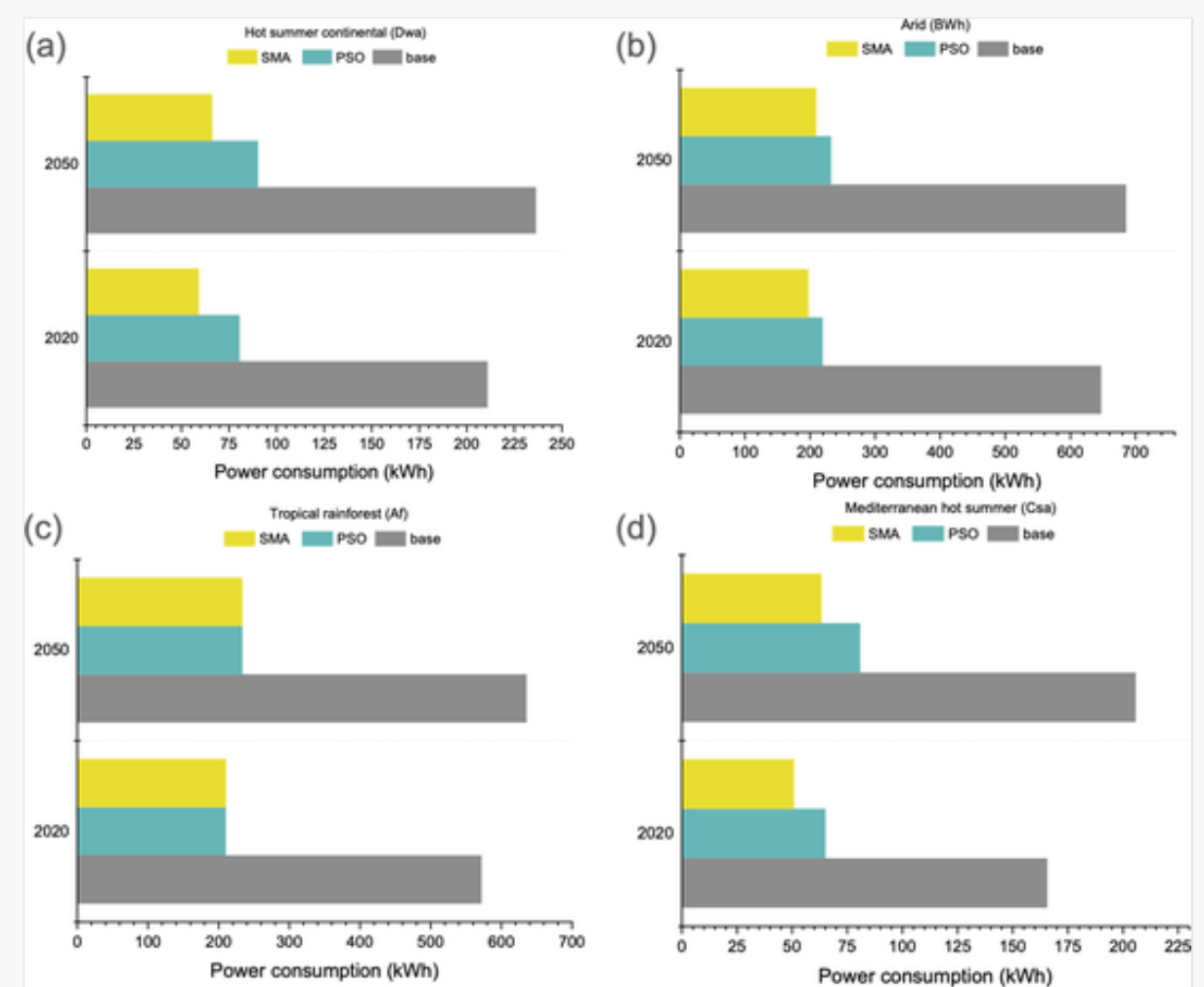

Annual power consumption in 2020 and 2050: (a): hot summer continental; (b): arid; (c): tropical rainforest; (d): Mediterranean hot summer.

In arid climate, as shown in Fig. 7 (b), the same story is predicted where the power consumption of the base system in 2020 with 5486 operating hours is $647.34(\mathrm{kWh})$ while PSO system consumes $66.10 \%$ less energy and the SMA consumes $69.49 \%$ less energy. In 2050 , the power consumption by the base system is estimated to be $685.69(\mathrm{kWh})$ while the PSO and SMA systems are estimated to consume $453.25(\mathrm{kWh})$ and $476.49(\mathrm{kWh})$ less power respectively.

Owing to the similar optimum operating and design parameters, the power consumption by both optimized systems in tropical rainforest climate is almost same. As seen in Fig. 7 (c), the power consumption by the base system in 2020 is $572.01(\mathrm{kWh})$ while $63.24 \%$ less power is consumed by optimized systems. In 2050 the power consumption by the base system is $635.75(\mathrm{kWh})$ while for the both optimized systems is $233.73(\mathrm{kWh})$.

As seen in Fig. 7 (d), in Mediterranean hot summer climate in 2020, the power consumption of the base system is estimated to be $165.67(\mathrm{kWh})$ whereas it has reduced to $65.13(\mathrm{kWh})$ and $50.83(\mathrm{kWh})$ for PSO and SMA systems respectively. In 2050, the base system is estimated to consume $205.80(\mathrm{kWh})$ power while PSO and SMA systems consume $80.91(\mathrm{kWh})$ and $63.25(\mathrm{kWh})$ power respectively.

The aforementioned explanations revealed that in general, the power consumption of all systems will increase by 2050 which is mainly due to the more operating hours caused by the global warming. Although this increase is valid for both optimized systems but the optimization has resulted in significant power saving.

\section{Conclusion}

The SHapley Additive exPlanations (SHAP) is used for the Guideless Irregular Dew Point Cooler (GIDPC) to illustrate the features contributions on the predicted parameters. In addition, two Evolutionary Optimisation (EO) algorithms, i.e., Slime Mould Algorithm (SMA) and Particle Swarm Optimization (PSO) are employed to optimise the system performance in 2020 and 2050. Four climates with one representative city for each are selected to compare the performance of the base system with optimized systems. The climate data revelaed that in general, the operating hours increase from 2020 to 2050 as a result of the global warming. However, it is possible to reduce the power consumption through the optimisation. The main outcomes of this reseach study is summarised as follows:

- The optimization results revealed that in general, performance of both optimized system in terms of COP outperform the base sysetem in all climates in both 2020 and 2050.

- The statistical analysis on the COP values revealed that, in tropical rainforest 2020 and 2050 both PSO 
and SMA have the same statistical distance from the base model whereas in hot summer continental and Arid climates, PSO results have larger statistical differences. But in Mediterranean hot summer the SMA results have a higher rate of statistical differences.

- The surface area of the optimized system by SMA's selected scenario (No.1) is same as the base system $62.49\left(\mathrm{~m}^{2}\right)$ in tropical rainforest climate whereas it has decreased to the values of $40.70\left(\mathrm{~m}^{2}\right), 44.99\left(\mathrm{~m}^{2}\right)$, and $51.19\left(\mathrm{~m}^{2}\right)$ in hot summer, arid and tropical Mediterranean climates. But in the optimized system by PSO, it has only decreased in the Arid climate, i.e., $53.78\left(\mathrm{~m}^{2}\right)$ and it is almost unchanged in all other climates.

- In spite of more operating hours in 2050, the power consumption of the optimized systems is estimated to substantially decrease. The power consumption of the base system in all climates was in the range of 165.67-647.34 $(\mathrm{kWh})$ while it has reduced to the ranges of 65.13-234.09 (kWh) and 50.83-209.46 $(\mathrm{kWh})$ by the PSO and SMA systems respectively. This has resulted in power savings of up to $72 \%$, $69.49 \%, 63.24 \%$, and $69.21 \%$ in hot summer continental, Arid, tropical rainforest and Mediterranean hot summer climates respectively.

\section{Future works}

In future works, one primary idea would be employing different mutation and acceleration mechanisms to improve the SMA performance. In addition, with the purpose of feature selection, the binary study based on the SMA. can be conducted. SMA can be a potential method to optimise the parameters of different ML methods such as SVM. Additionally, the advanced pareto-front optimisation can be developed for the new SMA method.

\section{Uncited references}

$[3,4,6,37]$.

CRediT authorship contribution statement ${ }_{\text {[nstruction: The authorship contribution is missing the }}$ following authors:

5th author: Ali Badiei: review and editing

6th author: Xin Xiao: review and editing

7th author: Samson Shittu: review and editing

]

Yousef Golizadeh Akhlaghi: Conceptualization, Investigation, Writing - original draft. Koorosh Aslansefat: Investigation, Conceptualization, Investigation, Conceptualization. Xudong Zhao: Project administration, Funding acquisition. Saba Sadati: Writing - original draft. Yi Fan: Investigation. Xiaoli Ma: Supervision.

\section{Declaration of Competing Interest}

The authors declare that they have no known competing financial interests or personal relationships that could have appeared to influence the work reported in this paper.

\section{Acknowledgement}

The authors would acknowledge our sincere appreciation to the financial supports from the European Commission H2020 MSCA programme (for the EU H2020-MSCA-RISE-2016-734340 DEW-COOL-4-CDC project), the National Key R\&D Program of China (Grant No. 2016YFE0133300) and UK BEIS project IEEA2021.

\section{References}

(i) The corrections made in this section will be reviewed and approved by a journal production editor. The newly added/removed references and its citations will be reordered and rearranged by the production team.

[1] Duan Z., Zhan C., Zhang X., Mustafa M., Zhao X., Alimohammadisagvand B., et al. Indirect evaporative cooling: Past, present and future potentials. Renew Sustain Energy Rev 2012;16:6823- 
[2] Pérez-Lombard L., Ortiz J., Pout C. A review on buildings energy consumption information. Energy Build 2008;40:394-398. doi:10.1016/j.enbuild.2007.03.007.

[3] Kabeel A.E., Abdelgaied M. Numerieal and experimental investigation of a novel configuration of indireet evaporative cooler with internal baffles. Energy Convers Manag 2016;126:526-536. dei:10.1016/j.eneonman.2016.08.028.

[4] Anisimov S., Pandelidis D., Danielewiez J. Numerieal analysis of selected evaporative exchangers with the Maisotsenko eyele. Energy Convers Manag 2014;88:426-441. dei:10.1016/j.eneonman.2014.08.055.

[5] Caliskan H, Dincer I, Hepbasli A. Exergoeconomic, enviroeconomic and sustainability analyses of a novel air cooler. Energy Build., vol. 55, Elsevier; 2012, p. 747-56. 10.1016/j.enbuild.2012.03.024.

[6] Chut K.J., Chou S.K., Yang W.M., Yan J. Achieving better energy-effieient air conditioning - A review of technologies and strategies. Appl Energy 2013;104:87 104. doi:10.1016/j.apenergy.2012.10.037.

[7] Company LE gas and electric. Laboratory Evaluation of the Coolerado cooler indirect evaporative cooling unit 2006.

[8] Maisotsenko V, Gillan L, ... TH-UP, 2003 undefined. Method and plate apparatus for dew point evaporative cooler; 2003.

[9] Jradi M., Riffat S. Experimental and numerical investigation of a dew-point cooling system for thermal comfort in buildings. Appl Energy 2014;132:524-535. doi:10.1016/j.apenergy.2014.07.040.

[10] Xu P., Ma X., Diallo T.M.O., Zhao X., Fancey K., Li D., et al. Numerical investigation of the energy performance of a guideless irregular heat and mass exchanger with corrugated heat transfer surface for dew point cooling. Energy 2016;109:803-817. doi:10.1016/j.energy.2016.05.062.

[11] Xu P., Ma X., Zhao X., Fancey K. Experimental investigation of a super performance dew point air cooler. Appl Energy 2017;203:761-777. doi:10.1016/j.apenergy.2017.06.095.

[12] Liu Y., Li J.M., Yang X., Zhao X. Two-dimensional numerical study of a heat and mass exchanger for a dew-point evaporative cooler. Energy 2019;168:975-988. doi:10.1016/j.energy.2018.11.135.

[13] Liu Y., Akhlaghi Y.G., Zhao X., Li J. Experimental and numerical investigation of a high-efficiency dew-point evaporative cooler. Energy Build 2019;197:120-130. doi:10.1016/j.enbuild.2019.05.038.

[14] Badiei A., Akhlaghi Y.G., Zhao X., Li J., Yi F., Wang Z. Can whole building energy models outperform numerical models, when forecasting performance of indirect evaporative cooling systems? Energy Convers Manag 2020;213. doi:10.1016/j.enconman.2020.112886.

[15] Mitchell T.M. Machine learning and data mining. Commun ACM 1999;42:30-36. doi: $10.1145 / 319382.319388$.

[16] Anandakumar H., Umamaheswari K. Supervised machine learning techniques in cognitive radio networks during cooperative spectrum handovers. Cluster Comput 2017;20:1505-1515. doi:10.1007/s10586-017-0798-3.

[17] Kang J.N., Wei Y.M., Liu L.C., Han R., Yu B.Y., Wang J.W. Energy systems for climate change mitigation: A systematic review 114602. Appl Energy 2020;263. doi:10.1016/j.apenergy.2020.114602.

[18] Iqbal M., Azam M., Naeem M., Khwaja A.S., Anpalagan A. Optimization classification, algorithms and tools for renewable energy: A review. Renew Sustain Energy Rev 2014;39:640-654. doi:10.1016/j.rser.2014.07.120. 
Liu Q., Li X., Liu H., Guo Z. Multi-objective metaheuristics for discrete optimization problems: A review of the state-of-the-art 106382. Appl Soft Comput J 2020;93. doi:10.1016/j.asoc.2020.106382.

[20] Pandelidis D., Anisimov S. Application of a statistical design for analyzing basic performance characteristics of the cross-flow Maisotsenko cycle heat exchanger. Int J Heat Mass Transf 2016;95:4561. doi:10.1016/j.ijheatmasstransfer.2015.11.060.

[21] Sohani A., Sayyaadi H., Hoseinpoori S. Modélisation et optimisation à objectifs multiples d'un refroidisseur évaporatif indirect à écoulements croisés à cycle $\mathrm{M}$ en utilisant le réseau neuronal de type GMDH. Int J Refrig 2016;69:186-204. doi:10.1016/j.jirefrig.2016.05.011.

[22] Sohani A., Sayyaadi H., Zeraatpisheh M. Optimization strategy by a general approach to enhance improving potential of dew-point evaporative coolers. Energy Convers Manag 2019;188:177-213. doi:10.1016/j.enconman.2019.02.079.

[23] Pakari A., Ghani S. Regression models for performance prediction of counter flow dew point evaporative cooling systems. Energy Convers Manag 2019;185:562-573. doi:10.1016/j.enconman.2019.02.025.

[24] Lin J., Wang R., Li C., Wang S., Long J., Chua K.J. Towards a thermodynamically favorable dew point evaporative cooler via optimization 112224. Energy Convers Manag 2020;203. doi:10.1016/j.enconman.2019.112224.

[25] Akhlaghi Y.G., Zhao X., Shittu S., Badiei A., Cattaneo M.E.G.V., Ma X. Statistical investigation of a dehumidification system performance using Gaussian process regression 202. Energy Build 2019. doi:10.1016/j.enbuild.2019.109406.

[26] Akhlaghi Y.G., Ma X., Zhao X., Shittu S., Li J. A statistical model for dew point air cooler based on the multiple polynomial regression approach 181. Energy 2019. doi:10.1016/j.energy.2019.05.213.

[27] Golizadeh Akhlaghi Y., Badiei A., Zhao X., Aslansefat K., Xiao X., Shittu S., et al. A constraint multiobjective evolutionary optimization of a state-of-the-art dew point cooler using digital twins. Energy Convers Manag 2020. doi:10.1016/j.enconman.2020.112772.

[28] Goodman B., Flaxman S. European union regulations on algorithmic decision making and a "right to explanation”. AI Mag 2017;38:50-57. doi:10.1609/aimag.v38i3.2741.

[29] World Map of the Köppen-Geiger climate classification updated: Ingenta Connect n.d.

[30] Atlanta AH-I., GA U, USA. American society of heating, refrigerating and air-conditioning engineers 2009.

[31] Chauvin Y, Rumelhart D. Backpropagation: theory, architectures, and applications; 1995.

[32] Gunning D. Explainable Artificial Intelligence (XAI); 2017.

[33] Preece A, Harborne D, Braines D, Tomsett R, Chakraborty S. Stakeholders in Explainable AI 2018.

[34] Barredo Arrieta A., Díaz-Rodríguez N., Del Ser J., Bennetot A., Tabik S., Barbado A., et al. Explainable Explainable Artificial Intelligence (XAI): Concepts, taxonomies, opportunities and challenges toward responsible AI. Inf Fusion 2020;58:82-115. doi:10.1016/j.inffus.2019.12.012.

[35] Lundberg SM, Allen PG, Lee S-I. A Unified Approach to Interpreting Model Predictions; 2020.

[36] Štrumbelj E., Kononenko I. Explaining prediction models and individual predictions with feature contributions. Knowl Inf Syst 2014;41:647-665. doi:10.1007/s10115-013-0679-x.

[37] Roth A. The Shapley value: essays in honor of Lloyd S. Shapley; 1988.

[38] Lundberg S, Nair B, Vavilala M, ... MH-N biomedical. Explainable machine-learning predictions for the prevention of hypoxaemia during surgery. NatureCom 2918. 
[39] Lin L, Gen M. Auto-tuning strategy for evolutionary algorithms: Balancing between exploration and exploitation. Soft Comput., vol. 13, Springer; 2009, p. 157-68. 10.1007/s00500-008-0303-2.

[40] Wolpert D, Evolutionary WM-I transactions on, 1997 U. No free lunch theorems for optimization. IeeexploreIeeeOrg 1997.

[41] Li S., Chen H., Wang M., Heidari A.A., Mirjalili S. Slime mould algorithm: A new method for stochastic optimization. Futur Gener Comput Syst 2020;111:300-323. doi:10.1016/j.future.2020.03.055.

[42] Kennedy, J RE-95-IC on N, 1995 U. Particle swarm optimization. IeeexploreIeeeOrg 1995.

[43] Zhang Y, Gong D, Sciences ZD-I. A bare-bones multi-objective particle swarm optimization algorithm for environmental/economic dispatch. Elsevier 2012.

[44] Zhang Y., Gong D.-W., Sun X.-Y., Geng N. Adaptive bare-bones particle swarm optimization algorithm and its convergence analysis. Springer 2014;18:1337-1352. doi:10.1007/s00500-013-1147-y.

[45] Song X, Zhang Y, Guo Y, ... XS. Variable-size Cooperative Coevolutionary Particle Swarm Optimization for Feature Selection on High-dimensional Data. IeeexploreIeeeOrg 2020.

[46] Gong D wei, Zhang Y, Qi C liang. Environmental/economic power dispatch using a hybrid multiobjective optimization algorithm. Int J Electr Power Energy Syst 2010;32:607-14. 10.1016/j.jepes.2009.11.017.

[47] Shi Y, Intelligence RE-IWC on C. A modified particle swarm optimizer, || in 1998 IEEE International Conference on Evolutionary Computation Proceedings 1998.

[48] Adopted I. Climate Change 2014 Synthesis Report 2014.

[49] Shen J., Copertaro B., Sangelantoni L., Zhang X., Suo H., Guan X. An early-stage analysis of climateadaptive designs for multi-family buildings under future climate scenario: Case studies in Rome, Italy and Stockholm, Sweden 100972. J Build Eng 2020;27. doi:10.1016/j.jobe.2019.100972.

[50] Picard T., Hong T., Luo N., Lee S.H., Sun K. Robustness of energy performance of Zero-Net-Energy (ZNE) homes 110251. Energy Build 2020;224. doi:10.1016/j.enbuild.2020.110251.

[51] Moazami A., Nik V.M., Carlucci S., Geving S. Impacts of future weather data typology on building energy performance - Investigating long-term patterns of climate change and extreme weather conditions. Appl Energy 2019;238:696-720. doi:10.1016/j.apenergy.2019.01.085.

\section{Footnotes}

\section{Text Footnotes}

[1] Correspondong authors: Xudong Zhao (email address: xudong.zhao@hull.ac.uk), Yousef Golizadeh Akhlaghi (email address: y.golizadeh-akhlaghi@hull.ac.uk)

\section{Highlights}

- Explainable Artificial Intelligence is used to interpret features contributions.

- New Slime Mould Algorithm is developed as the primary optimisation method.

- Particle Swarm Optimisation is considered as the comparing algorithm.

- Hourly weather data are produced using a high emission scenario in 2020 and 2050.

- Power savings of up to $72 \%$ is achievable by operation of optimized systems. 


\section{Queries and Answers}

\section{Q1}

Query: Your article is registered as a regular item and is being processed for inclusion in a regular issue of the journal. If this is NOT correct and your article belongs to a Special Issue/Collection please contact j.ramkumar@elsevier.com immediately prior to returning your corrections.

Answer: Yes

\section{Q2}

Query: The author names have been tagged as given names and surnames (surnames are highlighted in teal color). Please confirm if they have been identified correctly.

Answer: Yes

\section{Q3}

Query: Please note that as per standard style, a corresponding author footnote be provided for at least one author. Please check and assign the corresponding author name and email address.

Answer:

The corresponding author name and email address are as follows:

First name: Xudong

Surname: Zhao

Email: xudong.zhao@hull.ac.uk

Q4

Query: Uncited references: This section comprises references that occur in the reference list but not in the body of the text. Please cite each reference in the text or, alternatively, delete it. Any reference not dealt with will be retained in this section.

Answer: The four mentioned references are deleted. Actually they were removed from the context in the revision process but the list of references were not updated by mendeley software which we use.

So four mentioned references must be removed. 